\title{
Weak solutions for an initial-boundary Q-Tensor problem related to Liquid Crystals
}

\author{
F. GUILLÉN-GONZÁLEZ, \& M. A. RODRÍGUEZ-BELLIDO \\ Dpto. de Ecuaciones Diferenciales y Análisis Numérico, Universidad de Sevilla, Aptdo. 1160, \\ 41080 Sevilla, Spain. \\ guillen@us.es, angeles@us.es
}

\begin{abstract}
The coupled Navier-Stokes and Q-Tensor system is considered in a bounded three-dimensional domain under homogeneous Dirichlet boundary conditions for the velocity $\boldsymbol{u}$ and either nonhomogeneous Dirichlet or homogeneous Neumann boundary conditions for the tensor $Q$. The corresponding initial-value problem in the whole space $\mathbb{R}^{3}$ was analyzed in [Paicu \& Zarnescu, 2012].

In this paper, three main results concerning weak solutions will be proved; the existence of global in time weak solutions (bounded up to infinite time), a uniqueness criteria and a maximum principle for $Q$. Moreover, we identify how to modify the system to deduce symmetry and traceless for $Q$, for any weak solution. The presence of a stretching term in the $Q$-system plays a crucial role in all the analysis.
\end{abstract}

Keyword: Navier-Stokes equations, weak solution, uniqueness, maximum principle, symmetry, traceless

MSC codes: 35A01, 35A02, 35B50, 35D30, 35K51, 35Q35, 76A15, 76D03, 76D05

\section{The Q-Tensor system and main results}

\subsection{The model}

Liquid crystals can be seen as an intermediate phase of matter between crystalline solids and isotropic fluids. Nematic liquid crystals (N) consist of molecules with, for instance, rod-like shape whose center of mass is isotropically distributed and whose direction is almost constant on average over small regions. Several models of $(\mathrm{N})$ are described through the velocity and pressure $(\boldsymbol{u}, p)$ and a director vector denoted by $\boldsymbol{d}$ or $\boldsymbol{n}$ (cf. $[8,13]$ ).

The optical behaviour of liquid crystals can change from one point to the other and can be of different types, namely uniaxial (points having one single refractive index and a unitvector field $\boldsymbol{n}(\boldsymbol{x}) \in \mathbb{S}^{2}$ represents the preferred direction of molecular alignment), biaxial (points 
having two indices of refraction, with more than one preferred direction of molecular alignment) or isotropic (points where the orientation of molecules is equally distributed in all directions). The two main continuum theories for nematic liquid crystals (cf. $[3,6,8]$ ) are: the Oseen-Frank theory, restricted to uniaxial nematic liquid crystal materials; and the more general Landau-De Gennes theory, which can account the three types of optic for $(\mathrm{N})$ : uniaxial, biaxial and isotropic phases.

In the Landau-De Gennes theory, the director vector $\boldsymbol{d}$ appearing in the Oseen-Frank theory is replaced by a symmetric and traceless matrix $Q \in \mathbb{R}^{3 \times 3}$, known as the Q-tensor order parameter, which measures the deviation of the second moment tensor from its isotropic value. A nematic liquid crystal is said to be isotropic when $\mathrm{Q}=0$, uniaxial when the $\mathrm{Q}$-tensor has two equal non-zero eigenvalues and can be written in the special form:

$$
Q(\boldsymbol{x})=s\left(\boldsymbol{n}(\boldsymbol{x}) \otimes \boldsymbol{n}(\boldsymbol{x})-\frac{1}{d} \mathbb{I}\right) \quad \text { with } s \in \mathbb{R} \backslash\{0\}, \boldsymbol{n} \in \mathbb{S}^{2}
$$

and biaxial when $\mathrm{Q}$ has three different eigenvalues and can be represented as follows (see Proposition 1 in [14]):

$$
Q=s\left(\boldsymbol{n} \otimes \boldsymbol{n}-\frac{1}{d} \mathbb{I}\right)+r\left(\mathbf{m} \otimes \mathbf{m}-\frac{1}{d} \mathbb{I}\right) \quad s, r \in \mathbb{R} ; \boldsymbol{n}, \mathbf{m} \in \mathbb{S}^{2} .
$$

The definition of the $Q$-tensor is related to the second moment of a probability measure $\mu(\boldsymbol{x}, \cdot): \mathcal{L}\left(\mathbb{S}^{2}\right) \rightarrow[0,1]$ for each $\boldsymbol{x} \in \Omega$, being $\mathcal{L}\left(\mathbb{S}^{2}\right)$ the family of Lebesgue measurable sets on the unit sphere. For any $A \subset \mathbb{S}^{2}, \mu(\boldsymbol{x}, A)$ is the probability that the molecules with centre of mass in a very small neighborhood of the point $\boldsymbol{x} \in \Omega$ are pointing in a direction contained in $A$. This probability (cf. [21]) must satisfy $\mu(\boldsymbol{x}, A)=\mu(\boldsymbol{x},-A)$ in order to reproduce the socalled "head-to-tail" symmetry. As a consequence, the first moment of the probability measure vanishes, that is

$$
\langle p\rangle(\boldsymbol{x})=\int_{\mathbb{S}^{2}} p_{i} d \mu(\boldsymbol{x}, p)=0 .
$$

Then, the main information on $\mu$ comes from the second moment tensor

$$
M(\mu)_{i j}=\int_{\mathbb{S}^{2}} p_{i} p_{j} d \mu(p), \quad i, j=1,2,3
$$

It is easy to see that $M(\mu)=M(\mu)^{t}$ and $\operatorname{tr}(M)=1$. If the orientation of the molecules is equally distributed, then the distribution is isotropic and $\mu=\mu_{0}, d \mu_{0}(p)=\frac{1}{4 \pi} d A$ and $M\left(\mu_{0}\right)=\frac{1}{3} \mathbb{I}$. The deviation of the second moment tensor from its isotropic value is therefore measured as:

$$
Q=M(\mu)-M\left(\mu_{0}\right)=\int_{\mathbb{S}^{2}}\left(p \otimes p-\frac{1}{3} \mathbb{I}\right) d \mu(p) .
$$

From (1.1), $Q$ is symmetric and traceless. These properties are assumed (but not rigourously justified) in the problem studied by Paicu \& Zarnescu in [16] and Abels et al. in [1] (and in 
the more complete problem studied by the same authors in [15] and [2], respectively). These equations are also described in $[9,19]$ for fluids with constant density.

Now, firstly a generalization of the Q-tensor model given in [16] will be studied, and secondly some terms of this generic model will be rewritten appropriately to assure that any weak solution must be symmetric and traceless.

We are going to start studying a generic $Q$-tensor model in a smooth and bounded domain $\Omega \subset \mathbb{R}^{3}$, for the unknowns $(\boldsymbol{u}, p, Q):(0, T) \times \Omega \rightarrow \mathbb{R}^{3} \times \mathbb{R} \times \mathbb{R}^{3 \times 3}$, satisfying the momentum and incompressibility equations

$$
\left\{\begin{aligned}
D_{t} \boldsymbol{u}-\nu \Delta \boldsymbol{u}+\nabla p=\nabla \cdot \tau(Q)+\nabla \cdot \sigma(H, Q) & \text { in } \Omega \times(0, T), \\
\nabla \cdot \boldsymbol{u}=0 & \text { in } \Omega \times(0, T),
\end{aligned}\right.
$$

and the $Q$-tensor system:

$$
D_{t} Q-S(\nabla \boldsymbol{u}, Q)=-\gamma H(Q) \quad \text { in } \Omega \times(0, T)
$$

Here, $D_{t}=\partial_{t}+(\boldsymbol{u} \cdot \nabla)$ denotes the material time derivative, $\nu>0$ is the viscosity coefficient and $\gamma>0$ is a material-dependent elastic constant.

In (1.3), $S(\nabla \boldsymbol{u}, Q)=\nabla \boldsymbol{u} Q^{t}-Q^{t} \nabla \boldsymbol{u}$ is the so-called stretching term.

In (1.2) the tensors $\tau=\tau(Q)$ and $\sigma=\sigma(H, Q) \in \mathbb{R}^{3 \times 3}$ are defined by

$$
\left\{\begin{aligned}
\tau_{i j}(Q) & =-\varepsilon\left(\partial_{j} Q: \partial_{i} Q\right)=-\varepsilon \partial_{j} Q_{k l} \partial_{i} Q_{k l}, \varepsilon>0 \text { (symmetric tensor) } \\
\sigma(H, Q) & =H Q-Q H \text { (antisymmetric if } Q \text { and } H \text { are symmetric) }
\end{aligned}\right.
$$

where the tensor $H=H(Q)$ is related to the variational derivative in $L^{2}(\Omega)$ of a free energy functional $\mathcal{E}(Q)$, in fact

$$
H=\frac{\delta \mathcal{E}(Q)}{\delta Q}, \quad \mathcal{E}(Q)=\frac{\varepsilon}{2}|\nabla Q|^{2}+F(Q)
$$

Here, we denote $A: B=A_{i j} B_{i j}$ the scalar product of matrices (where the Einstein summation convention over repeated indices will be used) and the potential function $F(Q)$ is defined by

$$
F(Q)=\frac{a}{2}|Q|^{2}-\frac{b}{3}\left(Q^{2}: Q\right)+\frac{c}{4}|Q|^{4},
$$

with $a, b \in \mathbb{R}$ and $c>0$. We denote by $|Q|=(Q: Q)^{1 / 2}$ the matrix euclidean norm. Then, from (1.4) and (1.5)

$$
H=H(Q)=-\varepsilon \Delta Q+f(Q)
$$

where

$$
f(Q)=a Q-\frac{b}{3}\left(Q^{2}+Q Q^{t}+Q^{t} Q\right)+c|Q|^{2} Q
$$


Remark 1.1 It is easy to check that $f(Q)=\frac{\partial F}{\partial Q}(Q)$ using the equalities:

$$
\frac{\partial\left(Q^{2}: Q\right)}{\partial Q}=Q^{2}+Q Q^{t}+Q^{t} Q, \quad \frac{\partial\left(|Q|^{2}\right)}{\partial Q}=2 Q, \quad \frac{\partial\left(|Q|^{4}\right)}{\partial Q}=4|Q|^{2} Q .
$$

Finally, the system is completed with the following initial and boundary conditions over $\Gamma=\partial \Omega$ :

$$
\begin{gathered}
\left.\boldsymbol{u}\right|_{t=0}=\boldsymbol{u}_{0},\left.\quad Q\right|_{t=0}=Q_{0} \quad \text { in } \Omega \\
\left.\boldsymbol{u}\right|_{\Gamma}=\mathbf{0} \quad \text { in }(0, T),
\end{gathered}
$$

and

$$
\text { either }\left.\partial_{n} Q\right|_{\Gamma}=0 \quad \text { or }\left.\quad Q\right|_{\Gamma}=Q_{\Gamma} \quad \text { in }(0, T) \text {, }
$$

where $\boldsymbol{n}$ denotes the normal outwards vector on the boundary $\Gamma$.

Remark 1.2 The case of time-dependent Dirichlet boundary conditions:

$$
\left.Q\right|_{\Gamma}=Q_{\Gamma} \text { with } Q_{\Gamma}=Q_{\Gamma}(t)
$$

will be presented separately because its treatment is a little more technical (see Section 4).

\subsection{The main results of this paper}

We denote by $\boldsymbol{V}$ and $\boldsymbol{H}$ the solenoidal spaces:

$$
\boldsymbol{H}=\left\{\boldsymbol{v} \in \mathbf{L}^{2}(\Omega), \nabla \cdot \boldsymbol{v}=0,\left.\boldsymbol{v} \cdot \boldsymbol{n}\right|_{\partial \Omega}=0\right\}, \boldsymbol{V}=\left\{\boldsymbol{v} \in \mathbf{H}_{0}^{1}(\Omega), \nabla \cdot \boldsymbol{v}=0\right\}
$$

Now, we introduce the notion of weak solution we are going to handle with:

Definition 1.3 (Weak solution) It will be said that $(\boldsymbol{u}, Q)$ is a weak solution in $(0,+\infty)$ of problem (1.2)-(1.9) if:

$$
\left\{\begin{array}{l}
\boldsymbol{u} \in L^{\infty}(0,+\infty ; \mathbf{H}) \cap L^{2}(0,+\infty ; \mathbf{V}), \\
Q \in L^{\infty}\left(0,+\infty ; \mathbb{H}^{1}(\Omega)\right) \cap L^{2}\left(0 ; T ; \mathbb{H}^{2}(\Omega)\right), \quad \forall T>0
\end{array}\right.
$$

and satisfies the variational formulation (2.23) and (2.24) (defined below), the initial condition (1.7) and the boundary conditions (1.8)-(1.10).

Note that the regularity imposed in (1.11) is imposed up to infinite time excepting the $\mathbb{H}^{2}(\Omega)$-regularity for $Q$.

Now, we state the main results of this paper.

Theorem 1.4 (Existence of weak solution for time-independent b.c.) Assume $\left(\boldsymbol{u}_{0}, Q_{0}\right) \in$ $\boldsymbol{H} \times \mathbb{H}^{1}(\Omega)$ and $Q_{\Gamma} \in \mathbb{H}^{3 / 2}(\Gamma)$ for the case of time-independent boundary condition for $Q$. Then, there exists a weak solution $(\boldsymbol{u}, Q)$ of system (1.2)-(1.9) in $(0,+\infty)$. 
In order to study the time-dependent Dirichlet case imposed in (1.10), we need to lift the boundary data $Q_{\Gamma}(t)$, by using the "elliptic" problem

$$
-\Delta \widetilde{Q}(t)=0 \quad \text { in } \Omega, \quad \widetilde{Q}(t)=Q_{\Gamma}(t) \quad \text { on } \Gamma .
$$

Corollary 1.5 (Weak solution for time-dependent b.c.) Assume $\left(\boldsymbol{u}_{0}, Q_{0}\right) \in \boldsymbol{H} \times \mathbb{H}^{1}(\Omega)$ and the lifting function $\widetilde{Q}$ defined in (1.12) satisfies:

$$
\begin{aligned}
& \widetilde{Q} \in L^{\infty}\left(0,+\infty ; \mathbb{H}^{1}(\Omega)\right) \cap L^{2}\left(0, T ; \mathbb{H}^{2}(\Omega)\right), \\
& \partial_{t} \widetilde{Q} \in L^{4}\left(0, T ; \mathbb{L}^{4}(\Omega)\right) \cap L^{1}\left(0,+\infty ; \mathbb{L}^{1}(\Omega)\right) \cap L^{2}\left(0,+\infty ; \mathbb{L}^{2}(\Omega)\right),
\end{aligned}
$$

for all $T>0$, then there exists a weak solution $(\boldsymbol{u}, Q)$ of system (1.2)-(1.8) together with (1.10) in $(0,+\infty)$.

Remark 1.6 Owing to regularity of the Laplace problem (1.12), hypotheses (1.13) holds whenever

$$
Q_{\Gamma} \in L^{\infty}\left(0,+\infty ; \mathbb{H}^{1 / 2}(\Gamma)\right) \cap L^{2}\left(0, T ; \mathbb{H}^{3 / 2}(\Gamma)\right),
$$

while (1.14) is obtained for instance imposing

$$
\partial_{t} Q_{\Gamma} \in L^{4}\left(0, T ; \mathbb{H}^{1 / 2}(\Gamma)\right) \cap L^{1}\left(0,+\infty ; \mathbb{H}^{1 / 2}(\Gamma)\right) \cap L^{2}\left(0,+\infty ; \mathbb{H}^{1 / 2}(\Gamma)\right),
$$

although more general spaces could be considered by using the very-weak solution theory of problem (1.12).

Theorem 1.7 (Uniqueness criteria) Assume $\left(\boldsymbol{u}_{0}, Q_{0}\right) \in \boldsymbol{H} \times \mathbb{H}^{1}(\Omega)$. Let $(\boldsymbol{u}, Q)$ be a weak solution of system (1.2)-(1.9) in (0,T) (for a fixed $T>0$ ) such that $\nabla \boldsymbol{u}$ and $\Delta Q$ have the additional regularity:

$$
\begin{aligned}
& \nabla \boldsymbol{u} \in L^{\frac{2 q}{2 q-3}}\left(0, T ; \mathbf{L}^{q}(\Omega)\right) \quad \text { for some } q: 2 \leq q \leq 3, \\
& \Delta Q \in L^{\frac{2 s}{2 s-3}}\left(0, T ; \mathbb{L}^{s}(\Omega)\right) \quad \text { for some } s: 2 \leq s \leq 3 .
\end{aligned}
$$

Then, this solution coincides in $(0, T)$ with any weak solution associated to the same data.

Remark 1.8 Several remarks can be made on the uniqueness criteria (1.15):

- For the Navier-Stokes case (that is $Q=0$ ), Berselli proves in [4] that uniqueness criteria for $\nabla \boldsymbol{u}$ given in $(1.15)_{1}$ is also valid for $q>3 / 2$ and implies regularity and uniqueness. Now, we can only use it for $2 \leq q \leq 3$ (see the bound for the $I_{8}$-term in the proof of Theorem 1.7). A similar remark is true for the uniqueness criteria related to $\Delta Q$ given 
in $(1.15)_{2}$, which plays the role of the Berselli's regularity criteria for $Q$ and it is again only valid for $2 \leq s \leq 3$ (see the bound for the $I_{5}$-term in the proof of Theorem 1.7). In fact, this more restrictive situation is due to the stretching term $S(\nabla \boldsymbol{u}, Q)$ of the $Q$-system and the corresponding tensor $\sigma(H, Q)$ of the $\boldsymbol{u}$-system (see [12], where regularity criteria without these constraints on $q$ and/or $s$ are deduced for a nematic liquid crystal model without stretching).

- The alternative Serrin's regularity criteria for $\boldsymbol{u}, \boldsymbol{u} \in L^{\frac{2 r}{r-3}}\left(0, T ; \mathbf{L}^{r}(\Omega)\right)$ for some $r>3$ appearing in the Navier-Stokes case, and the corresponding for $\nabla Q$ in the same space, are not valid for this $Q$-tensor problem due to the presence of the stretching term (see again the bounds for the $I_{5}$ and $I_{8}$-terms in the proof of Theorem 1.7).

Theorem 1.9 (Maximum principle) Let $\alpha>0$ big enough, depending on the coefficients $(a, b, c)$ of the function $f(Q)$, such that

$$
\alpha^{2} \geq \frac{b^{2}}{c^{2}}-\frac{2 a}{c}
$$

Let $\boldsymbol{u} \in L^{2}(0, T ; \boldsymbol{V}) \cap L^{\infty}(0, T ; \boldsymbol{H})(0<T<+\infty)$ and $Q_{0} \in \mathbb{H}^{1}(\Omega)$ with $\left|Q_{0}(\boldsymbol{x})\right| \leq \alpha$ a.e. $\boldsymbol{x} \in \Omega$ (and $\left|Q_{\Gamma}(t, \boldsymbol{x})\right| \leq \alpha$ a.e. $(t, \boldsymbol{x}) \in(0, T) \times \partial \Omega$ in the case of Dirichlet boundary conditions for $Q)$. If $Q \in L^{\infty}\left(0, T ; \mathbb{H}^{1}(\Omega)\right) \cap L^{2}\left(0, T ; \mathbb{H}^{2}(\Omega)\right)$ is any point-wise solution for the Q-problem (1.3)-(1.7) $)_{2}$ in $(0, T) \times \Omega$ with boundary conditions (1.9) or (1.10), then

$$
|Q(t, \boldsymbol{x})| \leq \alpha \quad \text { a.e. }(t, \boldsymbol{x}) \in(0, T) \times \Omega .
$$

Note that the bound $\alpha$ is independent of the finite time $T$. Therefore, Theorem 1.9 gives the uniform bound $Q \in L^{\infty}\left(0,+\infty ; \mathbb{L}^{\infty}(\Omega)\right)$.

Remark 1.10 In [10,16] a maximum principle for a symmetric traceless matrix $Q$ satisfying the Cauchy problem related to (1.3) in the whole $\mathbb{R}^{3}$ is also proved. Concretely, a $L^{\infty}\left(0, T ; L^{\infty}\left(\mathbb{R}^{3}\right)\right)$ estimate is deduced from the inequality:

$$
\left(\int t^{p}\left(Q^{2}\right) d \boldsymbol{x}\right)^{1 /(2 p)} \leq e^{C T}\left(\int \operatorname{tr}^{p}\left(Q_{0}^{2}\right) d \boldsymbol{x}\right)^{1 /(2 p)}, \quad \forall p \in(1,+\infty)
$$

with $C$ independent of $p$. In particular, this maximum principle fails when $T \rightarrow+\infty$.

Remark 1.11 Theorems 1.4 and 1.7, which correspond to the time-independent boundary data, are proved without using the Maximum Principle for $Q$ given in Theorem 1.9. But in Corollary 1.5 (with time-dependent Dirichlet conditions for $Q$ ) the use of this maximum principle will be crucial to obtain the global in time weak regularity (see Subsection 4.3). Recently, we have known the work [2] where the weak regularity in $(0, T)$ for all $T>0$ for a more complete 
Q-tensor model than (1.2)-(1.9) (appearing in [15]) is analyzed (but the uniform estimates up to infinity time given in (1.11) are not considered in [2]). Again, in [2] $Q$ is already assumed symmetric and traceless and a non-homogeneous mixed Neumann/Dirichlet condition for $Q$ is considered. Now, we justifies rigorously the symmetry and traceless for $Q$ in Section 7, B and $C$, and we extend the weak existence given in [2] to the time-dependent Dirichlet case (although only the homogeneous Neumann case is treated).

Remark 1.12 The case of Nematic Liquid Crystal models with stretching terms via the OseenFrank theory, where the director vector $\boldsymbol{d} \in \mathbb{R}^{3}$ plays the role of the tensor $Q \in \mathbb{R}^{3 \times 3}$, could be studied following the same arguments of this work. One of these models has been analyzed for example in [18] and [20] but only for periodic boundary conditions for $(\boldsymbol{u}, \boldsymbol{d})$. Therefore, the existence of weak solutions for these nematic models with stretching terms can be also deduced considering boundary conditions (1.9) or (1.10) for $\boldsymbol{d}$. But, when time-dependent boundary data is considered for $\boldsymbol{d}$, since these models have not a maximum principle for $\boldsymbol{d}$, then we can only deduce the finite-time weak-regularity. On the other hand, the uniqueness criteria of Theorem 1.7 improves the criteria given in [20] assuming $(\boldsymbol{u}, \boldsymbol{d}) \in L^{\infty}\left(0, T ; \mathbf{H}^{1}(\Omega) \times \mathbb{H}^{2}(\Omega)\right)$.

In order to define a physically consistent model, the traceless and symmetry for the tensor $Q$ must be obtained. This is the case of the models studied by Paicu \& Zarnescu in $[15,16]$ and Abels et a. in $[1,2]$. The next results prove that changing the model (1.2)-(1.6) in an adequate manner and considering either traceless or symmetric initial and boundary data, such properties can be deduced for any weak solution of the model (although these constraints are not essential in the proof of existence, regularity and uniqueness results).

Theorem 1.13 (Traceless system) Consider the modified Q-tensor model defined by (1.2)(1.3)-(1.5) where the $H$-tensor given in (1.6) is replaced by:

$$
\widetilde{H}=H+\alpha(Q) \mathbb{I}
$$

where $\alpha(Q)$ is a scalar function defined by the convex combination

$$
\alpha(Q)=\lambda \alpha_{1}(Q)+(1-\lambda) \alpha_{2}(Q) \quad \text { for any } \lambda \in[0,1]
$$

with

$$
\alpha_{1}(Q):=\frac{1}{3}\left(-\operatorname{atr}(Q)+\frac{b}{3}\left(Q: Q^{t}+2|Q|^{2}\right)\right)
$$

and

$$
\alpha_{2}(Q):=-\frac{\operatorname{tr}(f(Q))}{3} .
$$

Assume the initial conditions (1.7) with $Q_{0}$ a traceless tensor, and the boundary conditions (1.8) for $\boldsymbol{u}$ and either (1.9) or (1.10) for $Q$ with traceless Dirichlet data $Q_{\Gamma}$. Then, for any weak solution $(\boldsymbol{u}, Q)$, one has that $Q(t)$ is a traceless tensor for any $t \geq 0$. 
Theorem 1.14 (Symmetric system) Consider the modified Q-tensor model defined by (1.2)(1.3)-(1.5) where the stretching term $S(\nabla \boldsymbol{u}, Q)$ is replaced by:

$$
\widehat{S}(\boldsymbol{W}, Q)=\boldsymbol{W} Q-Q \boldsymbol{W},
$$

$\boldsymbol{W}$ being the antisymmetric part of $\nabla \boldsymbol{u}, \boldsymbol{W}=\left(\nabla \boldsymbol{u}+(\nabla \boldsymbol{u})^{t}\right) / 2$. Impose the initial conditions (1.7) with $Q_{0}$ a symmetric tensor, and the boundary conditions (1.8) for $\boldsymbol{u}$ and either (1.9) or (1.10) for the tensor $Q$ with symmetric Dirichlet data $Q_{\Gamma}$. Then, for any weak solution $(\boldsymbol{u}, Q)$, one has that $Q(t)$ is a symmetric tensor for any $t \geq 0$.

From the previous results, the modified problem considering both traceless and symmetry can be written as follows (which coincides with the one studied by Paicu \& Zarnescu in [16] in the whole space $\mathbb{R}^{3}$ ):

$$
\left\{\begin{array}{r}
D_{t} \boldsymbol{u}-\nu \Delta \boldsymbol{u}+\nabla p=\nabla \cdot \tau(Q)+\nabla \cdot \sigma\left(H_{p z}, Q\right) \quad \text { in } \Omega \times(0, T), \\
\nabla \cdot \boldsymbol{u}=0 \quad \text { in } \Omega \times(0, T), \\
D_{t} Q-\widehat{S}(\boldsymbol{W}, Q)=-\gamma H_{p z}(Q) \quad \text { in } \Omega \times(0, T), \\
\left.\boldsymbol{u}\right|_{t=0}=\boldsymbol{u}_{0},\left.\quad Q\right|_{t=0}=Q_{0} \quad \text { in } \Omega, \\
\left.\boldsymbol{u}\right|_{\Gamma}=\mathbf{0} \quad \text { in }(0, T), \\
\text { either }\left.\partial_{n} Q\right|_{\Gamma}=0 \quad \text { or }\left.\quad Q\right|_{\Gamma}=Q_{\Gamma} \quad \text { in }(0, T),
\end{array}\right.
$$

where

$$
H_{p z}(Q)=-\varepsilon \Delta Q+a Q-b\left(Q^{2}-\frac{\operatorname{tr}\left(Q^{2}\right)}{3} \mathbb{I}\right)+c|Q|^{2} Q
$$

with $\mathbb{I}$ the identity matrix in $\mathbb{R}^{3 \times 3}$, and the stretching term $\widehat{S}(\boldsymbol{W}, Q)$ is given by (1.20). In particular, Theorems 1.4, 1.7 and 1.9 and Corollary 1.5 can be extended to this traceless and symmetric problem (1.21).

On the other hand, some local in time strong regularity results for problem (1.21) have been recently deduced in $[1,11]$, but only imposing homogeneous Dirichlet condition for $Q$.

The rest of the paper is organized as follows. In Section 2 we give the variational formulation of the problem. Afterwards, we will obtain the energy law and prove the existence of weak solutions; Theorem 1.4 in Section 3 and Corollary 1.5 in Section 4. Section 5 is devoted to the search of uniqueness criteria for weak solutions (Theorem 1.7). The maximum principle will be proved in Section 6 (Theorem 1.9). In Section 7, some terms in the model (1.2)-(1.3) are modified in order to obtain the traceless of $Q$ (Theorem 1.13) and symmetry of $Q$ (Theorem 1.14), for any weak solution $(\boldsymbol{u}, Q)$. The extension of Theorems 1.4, 1.7 and 1.9 and Corollary 1.5 to these 
modified problems are analyzed in B and C, corresponding to the constraints of traceless and symmetry, respectively. The existence of Galerkin weak solutions, without or with traceless and symmetry restrictions, are proved in $\mathrm{A}$ and $\mathrm{D}$, respectively.

\section{Variational formulation}

Taking into account that $\partial_{i} F(Q)=F^{\prime}(Q): \partial_{i} Q=f(Q): \partial_{i} Q$, the term of the symmetric tensor $\tau$ can be rewritten as:

$$
\begin{aligned}
(\nabla \cdot \tau)_{i} & =-\varepsilon \partial_{j}\left(\partial_{j} Q: \partial_{i} Q\right)=-\varepsilon \Delta Q: \partial_{i} Q-\varepsilon \partial_{j} Q: \partial_{i j}^{2} Q \\
& =H(Q): \partial_{i} Q-\partial_{i}\left(F(Q)+\frac{\varepsilon}{2}|\nabla Q|^{2}\right),
\end{aligned}
$$

where $|\nabla Q|^{2}=\partial_{j} Q: \partial_{j} Q$. Then, testing (1.2) by any $\overline{\boldsymbol{u}}: \Omega \rightarrow \mathbb{R}^{3}$ with $\left.\overline{\boldsymbol{u}}\right|_{\partial \Omega}=\mathbf{0}$ and $\nabla \cdot \overline{\boldsymbol{u}}=0$ in $\Omega$, we arrive at the following variational formulation of (1.2):

$$
\left(D_{t} \boldsymbol{u}, \overline{\boldsymbol{u}}\right)+\nu(\nabla \boldsymbol{u}, \nabla \overline{\boldsymbol{u}})-((\overline{\boldsymbol{u}} \cdot \nabla) Q, H)+(\sigma(H, Q), \nabla \overline{\boldsymbol{u}})=0 .
$$

On the other hand, testing (1.3) by $\bar{H}$ and the system $-\varepsilon \Delta Q+f(Q)=H$ by $\bar{Q}$, we arrive at the variational formulation:

$$
\left\{\begin{aligned}
\left(\partial_{t} Q, \bar{H}\right)+((\boldsymbol{u} \cdot \nabla) Q, \bar{H})-(S(\nabla \boldsymbol{u}, Q), \bar{H})+\gamma(H, \bar{H}) & =0 \\
\varepsilon(\nabla Q, \nabla \bar{Q})+(f(Q), \bar{Q})-(H, \bar{Q}) & =0
\end{aligned}\right.
$$

for any $\bar{H}$ and $\bar{Q}: \Omega \rightarrow \mathbb{R}^{3 \times 3}$ with $\left.\bar{Q}\right|_{\partial \Omega}=0$ in the case of Dirichlet data for $Q$. From (2.24), one has in particular:

$$
\left(\partial_{t} Q, \bar{Q}\right)+((\boldsymbol{u} \cdot \nabla) Q, \bar{Q})-\varepsilon \gamma(\Delta Q, \bar{Q})+\gamma(f(Q), \bar{Q})=0
$$

Remark 2.1 By using the weak regularity (1.11) and the following interpolation inequalities:

$$
\|Q\|_{\mathbb{L}^{\infty}(\Omega)} \leq\|Q\|_{\mathbb{H}^{1}(\Omega)}^{1 / 2}\|Q\|_{\mathbb{H}^{2}(\Omega)}^{1 / 2}, \quad\|v\|_{\mathbf{L}^{3}(\Omega)} \leq\|v\|_{L^{2}(\Omega)}^{1 / 2}\|v\|_{H^{1}(\Omega)}^{1 / 2},
$$

then (2.25) implies that the $Q$-system (1.3) is satisfied point-wisely a.e. in $(0,+\infty) \times \Omega$, in fact

$$
\partial_{t} Q+(\boldsymbol{u} \cdot \nabla) Q-S(\nabla \boldsymbol{u}, Q)+\gamma(-\varepsilon \Delta Q+f(Q))=0 \quad \text { in } L^{4 / 3}\left(0, T ; \mathbb{L}^{2}(\Omega)\right),
$$

for any $T>0$. On the other hand, (2.23) implies that the $\boldsymbol{u}$-system (1.2) is satisfied variationally as

$$
\partial_{t} \boldsymbol{u}+(\boldsymbol{u} \cdot \nabla) \boldsymbol{u}-\nu \Delta \boldsymbol{u}-\nabla \cdot(\nabla Q \otimes \nabla Q+(H Q-Q H))=0 \quad \text { in } L^{4 / 3}\left(0, T ; \mathbf{V}^{\prime}\right) .
$$

In particular, the variational formulation (2.23) and (2.25) have sense for test functions $\overline{\boldsymbol{u}} \in$ $L^{4}(0, T ; \mathbf{V})$ and $\bar{Q} \in L^{4}\left(0, T ; \mathbb{L}^{2}(\Omega)\right)$, respectively.

On the other hand, $Q \in C\left([0, T] ; \mathbb{L}^{2}(\Omega)\right)$ and $\boldsymbol{u} \in C\left([0, T] ; \mathbf{V}^{\prime}\right)$ hence the initial conditions (1.7) have sense. 
Remark 2.2 The regularity for the pressure $p$ can be deduced from De Rham's Theorem, obtaining $p \in W^{-1, \infty}\left(0, T ; L^{2}(\Omega)\right)$ in a similar way to the Navier-Stokes system, because of the tensors terms $\nabla \cdot \tau(Q)$ and $\nabla \cdot \sigma(H, Q)$ have the same regularity as $(\boldsymbol{u} \cdot \nabla) \boldsymbol{u}=\nabla \cdot(\boldsymbol{u} \otimes \boldsymbol{u})$.

\section{Existence of weak solutions (proof of Theorem 1.4)}

The proof of the existence of weak solutions is based on three main ingredients:

- Formal a priori estimates (see Subsection 3.1). These a priori estimates are going to be justified via a Galerkin approximation.

- Existence of Galerkin solutions (see A).

- Passage to the limit, using compactness results (see Subsection 3.2).

\subsection{Dissipative energy law and global in time a priori estimates}

Taking $\bar{Q}=\partial_{t} Q$ in $(2.24)_{2}$ and considering homogeneous Neumann or time-independent Dirichlet boundary conditions for $Q$, it holds:

$$
\begin{aligned}
\left(H, \partial_{t} Q\right) & =\varepsilon\left(\nabla\left(\partial_{t} Q\right), \nabla Q\right)+\left(\partial_{t} Q, F^{\prime}(Q)\right) \\
& =\frac{d}{d t}\left(\frac{\varepsilon}{2}\|\nabla Q\|_{\mathbb{L}^{2}(\Omega)}^{2}+\int_{\Omega} F(Q) d \boldsymbol{x}\right)=\frac{d}{d t} \int_{\Omega} \mathcal{E}(Q) d \boldsymbol{x}
\end{aligned}
$$

where $\mathcal{E}(Q)$ is defined in (1.4).

Now, we want to obtain an "energy equality". With this objective, it will be of great help the following Lemma:

Lemma 3.1 For any $A, B, C \in \mathbb{R}^{n \times n}$, the following identities hold:

$$
A B: C=B: A^{t} C \quad \text { and } \quad A: B C=A C^{t}: B .
$$

It allows to obtain that $S(\nabla \boldsymbol{u}, Q): \bar{H}=\sigma(\bar{H}, Q): \nabla \boldsymbol{u}$. Indeed,

$$
S(\nabla \boldsymbol{u}, Q): \bar{H}=\left(\nabla \boldsymbol{u} Q^{t}-Q^{t} \nabla \boldsymbol{u}\right): \bar{H}=\nabla \boldsymbol{u}: \bar{H} Q-\nabla \boldsymbol{u}: Q \bar{H}=\nabla \boldsymbol{u}: \sigma(\bar{H}, Q) .
$$

As a consequence, taking $\overline{\boldsymbol{u}}=\boldsymbol{u}$ in $(2.23)$ and $(\bar{H}, \bar{Q})=\left(H, \partial_{t} Q\right)$ in $(2.24)$, then the stretching term cancels with the term dependent on the tensor $\sigma(H, Q)$, the term $((\boldsymbol{u} \cdot \nabla) Q, H)$ appearing in both (2.23) and (2.24) also cancels and the convection term $((\boldsymbol{u} \cdot \nabla) \boldsymbol{u}, \boldsymbol{u})$ vanishes, hence the following "energy equality" holds:

$$
\frac{d}{d t}\left(\frac{1}{2}\|\boldsymbol{u}\|_{\mathbf{L}^{2}(\Omega)}^{2}+\int_{\Omega} \mathcal{E}(Q) d \boldsymbol{x}\right)+\nu\|\nabla \boldsymbol{u}\|_{\mathbf{L}^{2}(\Omega)}^{2}+\gamma\|H\|_{\mathbb{L}^{2}(\Omega)}^{2}=0 .
$$


Regularity bounds for $(\boldsymbol{u}, Q)$ is not guaranteed from $(3.26)$ yet, because of $\int_{\Omega} \mathcal{E}(Q) d \boldsymbol{x}$ is not a positive term due to $F(Q)$. Next, we see that a new positive potential function (up to an additive constant) can replace $F(Q)$. By using the following bounds; there exists some constants $\alpha_{1}=\alpha_{1}(b, c), \alpha_{2}=\alpha_{2}(b, c), \beta(a, c)>0$ such that

$$
\begin{gathered}
\left|\frac{b}{3} Q^{2}: Q\right| \leq \frac{|b|}{3}|Q|^{2}|Q| \leq \begin{cases}\frac{c}{8}|Q|^{4}+\alpha_{1}(b, c), & \text { if } a \geq 0, \\
\frac{c}{16}|Q|^{4}+\alpha_{2}(b, c), & \text { if } a<0,\end{cases} \\
\left.\left.\left|\frac{a}{2}\right| Q\right|^{2}\left|\leq \frac{c}{16}\right| Q\right|^{4}+\beta(a, c),
\end{gathered}
$$

the following lower bound of $F(Q)$ holds:

$$
F(Q) \geq \begin{cases}\frac{a}{2}|Q|^{2}+\frac{c}{8}|Q|^{4}-\alpha_{1}, & \text { if } a>0, \\ \frac{c}{8}|Q|^{4}-\alpha_{2}-\beta, & \text { if } a<0 .\end{cases}
$$

As a consequence, we can define

$$
F_{\mu}(Q)=F(Q)+\mu
$$

with $\mu=\alpha_{1}$ if $a \geq 0$ and $\mu=\alpha_{2}+\beta$ if $a<0$, such that:

$$
F_{\mu}(Q) \geq \begin{cases}\frac{a}{2}|Q|^{2}+\frac{c}{8}|Q|^{4} \geq 0 & \text { if } a>0, \\ \frac{c}{8}|Q|^{4} \geq 0 & \text { if } a<0 .\end{cases}
$$

In both cases,

$$
F_{\mu}(Q) \geq \frac{c}{8}|Q|^{4}
$$

Therefore, replacing in $(3.26) \mathcal{E}(Q)$ by

$$
\mathcal{E}_{\mu}(Q)=\frac{1}{2}|\nabla Q|^{2}+F_{\mu}(Q) \geq 0
$$

we will obtain some global in time estimates (until infinite time). Indeed, assuming finite total energy of initial data, i.e.

$$
\int_{\Omega} \mathcal{E}_{\mu}\left(Q_{0}\right) d \boldsymbol{x}+\frac{1}{2}\left\|\boldsymbol{u}_{0}\right\|_{\mathbf{L}^{2}(\Omega)}^{2}<+\infty
$$

(here, $|\Omega|<+\infty$ is essential due to $\int_{\Omega} F_{\mu}\left(Q_{0}\right) d \boldsymbol{x}=\int_{\Omega} F\left(Q_{0}\right) d \boldsymbol{x}+\mu|\Omega|$ ), then the following estimates hold:

$$
\left\{\begin{aligned}
\boldsymbol{u} & \in L^{\infty}\left(0,+\infty ; L^{2}(\Omega)\right) \cap L^{2}\left(0,+\infty ; \mathbf{H}^{1}(\Omega)\right), \\
\nabla Q & \in L^{\infty}\left(0,+\infty ; \mathbb{L}^{2}(\Omega)\right), \\
H & \in L^{2}\left(0,+\infty ; \mathbb{L}^{2}(\Omega)\right), \\
F_{\mu}(Q) & \in L^{\infty}\left(0,+\infty ; L^{1}(\Omega)\right) .
\end{aligned}\right.
$$


In particular, from (3.27) and (3.28), we deduce the bounds:

$$
Q \in L^{\infty}\left(0,+\infty ; \mathbb{L}^{4}(\Omega)\right) \text { and } Q \in L^{\infty}\left(0,+\infty ; \mathbb{H}^{1}(\Omega)\right)
$$

hence, in particular

$$
Q \in L^{\infty}\left(0,+\infty ; \mathbb{L}^{6}(\Omega)\right)
$$

Since $f(Q)$ is a third order polynomial function,

$$
|f(Q)| \leq C(a, b, c)\left(|Q|+|Q|^{2}+|Q|^{3}\right)
$$

which together to $(3.29)$ gives $f(Q) \in L^{\infty}\left(0,+\infty ; \mathbb{L}^{2}(\Omega)\right)$. Then, using that $H(Q)=-\varepsilon \Delta Q+$ $f(Q)$, we obtain:

$$
\Delta Q \in L^{\infty}\left(0+\infty ; \mathbb{L}^{2}(\Omega)\right)+L^{2}\left(0,+\infty ; \mathbb{L}^{2}(\Omega)\right) \hookrightarrow L^{2}\left(0, T ; \mathbb{L}^{2}(\Omega)\right) \quad \forall T>0
$$

Finally, by using the $H^{2}$-regularity of the Poisson problem:

$$
\left\{\begin{aligned}
-\Delta Q+Q & =f \text { in } \Omega, \\
\left.\partial_{n} Q\right|_{\Gamma} & =0 \text { or }\left.Q\right|_{\Gamma}=Q_{\Gamma}
\end{aligned}\right.
$$

(the boundary data $Q_{\Gamma} \in \mathbb{H}^{3 / 2}(\Gamma)$ in the second case), we deduce that:

$$
Q \in L^{2}\left(0, T ; \mathbb{H}^{2}(\Omega)\right) \quad \forall T>0
$$

\subsection{Passage to the limit in $(0, T)$, for each $T>0$.}

The previous a priori estimates given in Subsection 3.1 can be justified via a Galerkin approximation $\left(\boldsymbol{u}^{m}, Q^{n}\right)$ defined in A. Then, a weak solution of problem (1.2)-(1.9) can be obtained passing to the limit as $(m, n) \uparrow+\infty$ in these Galerkin approximations. A similar argument is used in [7] for a nematic model in $(\boldsymbol{u}, \boldsymbol{d})$. In [5] a different Galerkin approximation is considered, where the $Q$-system is maintained at infinity dimension, adding a regularized viscous tensor of $p$-laplacian type.

Indeed, since the Galerkin approximations $\left(\boldsymbol{u}^{m}, Q^{n}\right)$ satisfy all the bounds of Subsections 3.1 (and Section 4 below for the time-dependent Dirichlet case), there exists a subsequence of $\left(\boldsymbol{u}^{m}, Q^{n}, H^{n}\right)$ (equally denoted for simplicity) and limit functions $(\boldsymbol{u}, Q, H)$ such that:

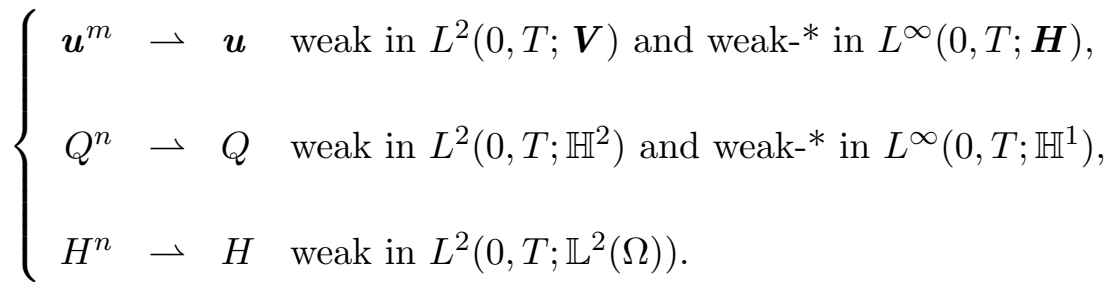


By using interpolation and product inequalities (see Remark 2.1), one has the following bounds in $L^{4 / 3}\left(0, T ; \mathbb{L}^{2}(\Omega)\right)$ :

$$
\boldsymbol{u}^{m} \otimes \boldsymbol{u}^{m}, \nabla Q^{n} \otimes \nabla Q^{n}, H^{n} Q^{n},\left(\boldsymbol{u}^{m} \cdot \nabla\right) Q^{n}, \nabla \boldsymbol{u}^{m} Q^{n}
$$

and

$$
f\left(Q^{n}\right) \text { in } L^{\infty}\left(0, T ; \mathbb{L}^{2}(\Omega)\right) \cap L^{2}\left(0, T ; \mathbb{H}^{1}(\Omega)\right) .
$$

In particular, by looking at the Galerkin problem, fixed $m_{0}, n_{0} \geq 0$ the following bounds for the time derivate functions hold:

$$
\left(\partial_{t} \boldsymbol{u}^{m}, \partial_{t} Q^{n}\right) \text { in } L^{4 / 3}\left(0, T ;\left(\boldsymbol{V}^{m_{0}}\right)^{\prime} \times\left(\mathbb{M}^{n_{0}}\right)^{\prime}\right)
$$

for any $m \geq m_{0}$ and $n \geq n_{0}$. Then, some compactness results can be applied (see [17]), obtaining the strong convergences:

$$
\begin{gathered}
\boldsymbol{u}^{m} \rightarrow \boldsymbol{u} \quad \text { in } L^{2}\left(0, T ; \mathbf{L}^{2}(\Omega)\right) \cap C\left([0, T] ; \boldsymbol{V}^{\prime}\right), \\
Q^{n} \rightarrow Q \quad \text { in } L^{2}\left(0, T ; \mathbb{H}^{1}(\Omega)\right) \cap C\left([0, T] ; \mathbb{L}^{2}(\Omega)\right) .
\end{gathered}
$$

In particular, by using (3.31), (3.32), (3.34) and (3.35), we have the following weak convergences in $L^{4 / 3}\left(0, T ; \mathbb{L}^{2}(\Omega)\right)$ for the nonlinear terms:

$$
\begin{aligned}
\boldsymbol{u}^{m} \otimes \boldsymbol{u}^{m} & \rightarrow \boldsymbol{u} \otimes \boldsymbol{u} \\
\nabla Q^{n} \otimes \nabla Q^{n} & \rightarrow \nabla Q \otimes \nabla Q \\
\left(\boldsymbol{u}^{m} \cdot \nabla\right) Q^{n} & \rightarrow(\boldsymbol{u} \cdot \nabla) Q \\
H^{n} Q^{n} & \rightarrow H Q \\
\nabla \boldsymbol{u}^{m} Q^{n} & \rightarrow \nabla \boldsymbol{u} Q .
\end{aligned}
$$

Interpolation results allow to deduce from (3.31) that

$$
Q^{n} \quad \text { is bounded in } \mathbb{L}^{10}((0, T) \times \Omega) \text {. }
$$

Hence, compactness of (3.35) can be extended to

$$
Q^{n} \rightarrow Q \quad \text { in } L^{p}\left(0, T ; \mathbb{L}^{p}(\Omega)\right) \text { for any } p<10
$$

Moreover, the Dominated Convergence Theorem leads to:

$$
g\left(Q^{n}\right) \rightarrow g(Q) \quad \text { in } L^{1}\left(0, T ; \mathbb{L}^{1}(\Omega)\right)
$$


for any continuous function $g$ whose increasing is dominated by $|g(Q)| \leq c_{1}|Q|^{p}+c_{2}$. In particular, since $f(Q)$ is dominated for $p=3$, one has

$$
f\left(Q^{n}\right) \rightarrow f(Q) \quad \text { in } L^{1}\left(0, T ; \mathbb{L}^{1}(\Omega)\right) .
$$

Moreover, again by using interpolation results, we can deduce that:

$$
f\left(Q^{n}\right) \rightarrow f(Q) \quad \text { weak-* }^{*} \text { in } L^{\infty}\left(0, T ; \mathbb{L}^{2}(\Omega)\right) \text { and weak in } L^{2}\left(0, T ; \mathbb{H}^{1}(\Omega)\right)
$$

As a consequence of previous convergences, first taking limit of the Galerkin approximations as $(m, n) \uparrow+\infty$ and afterwards in the test functions as $\left(m_{0}, n_{0}\right) \uparrow+\infty$, then the limit $(\boldsymbol{u}, Q, H)$ satisfies the variational formulation (2.23)-(2.24).

\section{Weak solutions for time-dependent Dirichlet data for $Q$ (proof of Corollary 1.5)}

\subsection{Finite time estimates.}

Defining $\widehat{Q}(t)=Q(t)-\widetilde{Q}(t)$, where $\widetilde{Q}(t)$ is the lifting function defined in (1.12), the following modified energy equality holds instead of (3.26):

$$
\begin{aligned}
\frac{d}{d t}\left(\frac{1}{2}\|\boldsymbol{u}\|_{\mathbf{L}^{2}(\Omega)}^{2}\right. & \left.+\int_{\Omega} \widehat{\mathcal{E}}(Q) d \boldsymbol{x}\right)+\nu\|\nabla \boldsymbol{u}\|_{\mathbf{L}^{2}(\Omega)}^{2} \\
& +\gamma\|-\varepsilon \Delta \widehat{Q}+f(Q)\|_{\mathbb{L}^{2}(\Omega)}^{2}=-\left(\partial_{t} \widetilde{Q},-\varepsilon \Delta \widehat{Q}\right)
\end{aligned}
$$

where $\int_{\Omega} \widehat{\mathcal{E}}(Q) d \boldsymbol{x}=\frac{\varepsilon}{2}\|\nabla \widehat{Q}\|_{\mathbb{L}^{2}(\Omega}^{2}+\int_{\Omega} F_{\mu}(Q) d \boldsymbol{x}$. Note that a "source term" appears at RHS of (4.36), that can be rewritten as:

$$
-\left(\partial_{t} \widetilde{Q},-\varepsilon \Delta \widehat{Q}\right)=-\left(\partial_{t} \widetilde{Q},-\varepsilon \Delta \widehat{Q}+f(Q)\right)+\left(\partial_{t} \widetilde{Q}, f(Q)\right) .
$$

The first term at RHS of (4.37) can be bounded as:

$$
\left|-\left(\partial_{t} \widetilde{Q},-\varepsilon \Delta \widehat{Q}+f(Q)\right)\right| \leq \frac{\gamma}{2}\|-\varepsilon \Delta \widehat{Q}+f(Q)\|_{\mathbb{L}^{2}(\Omega)}^{2}+\frac{1}{2 \gamma}\left\|\partial_{t} \widetilde{Q}\right\|_{\mathbb{L}^{2}(\Omega)}^{2} .
$$

Making use of the lower estimate for $F_{\mu}(Q)$ given in (3.27), it is easy to prove that the second term at RHS of (4.37) (depending on $\partial_{t} \widetilde{Q}$ ) is bounded as:

$$
\begin{aligned}
& \left|\left(\partial_{t} \widetilde{Q}, f(Q)\right)\right| \leq \int_{\Omega}\left(|a||Q|+|b||Q|^{2}+c|Q|^{3}\right)\left|\partial_{t} \widetilde{Q}\right| d \boldsymbol{x} \\
& \quad \leq \frac{c}{8} \int_{\Omega}|Q|^{4} d \boldsymbol{x}+C_{a, b, c}\left(\left\|\partial_{t} \widetilde{Q}\right\|_{\mathbb{L}^{4 / 3}(\Omega)}^{4 / 3}+\left\|\partial_{t} \widetilde{Q}\right\|_{\mathbb{L}^{2}(\Omega)}^{2}+\left\|\partial_{t} \widetilde{Q}\right\|_{\mathbb{L}^{4}(\Omega)}^{4}\right) \\
& \quad \leq \int_{\Omega} F_{\mu}(Q) d \boldsymbol{x}+C\left(\left\|\partial_{t} \widetilde{Q}\right\|_{\mathbb{L}^{4 / 3}(\Omega)}^{4 / 3}+\left\|\partial_{t} \widetilde{Q}\right\|_{\mathbb{L}^{2}(\Omega)}^{2}+\left\|\partial_{t} \widetilde{Q}\right\|_{\mathbb{L}^{4}(\Omega)}^{4}\right) .
\end{aligned}
$$


In such a way, from (4.36) we arrive at the following inequality:

$$
\begin{aligned}
& \frac{d}{d t}\left(\frac{1}{2}\|\boldsymbol{u}\|_{\mathbf{L}^{2}(\Omega)}^{2}+\frac{\varepsilon}{2}\|\nabla \widehat{Q}\|_{\mathbb{L}^{2}(\Omega}^{2}+\int_{\Omega} F_{\mu}(Q) d \boldsymbol{x}\right) \\
& \quad+\nu\|\nabla \boldsymbol{u}\|_{\mathbf{L}^{2}(\Omega)}^{2}+\frac{\gamma}{2}\|-\varepsilon \Delta \widehat{Q}+f(Q)\|_{\mathbb{L}^{2}(\Omega)}^{2} \\
& \quad \leq \int_{\Omega} F_{\mu}(Q) d \boldsymbol{x}+C_{\gamma}\left(\left\|\partial_{t} \widetilde{Q}\right\|_{\mathbb{L}^{4 / 3}(\Omega)}^{4 / 3}+\left\|\partial_{t} \widetilde{Q}\right\|_{\mathbb{L}^{2}(\Omega)}^{2}+\left\|\partial_{t} \widetilde{Q}\right\|_{\mathbb{L}^{4}(\Omega)}^{4}\right) .
\end{aligned}
$$

In this case, by using the Gronwall Lemma and assuming $\partial_{t} \widetilde{Q} \in L^{4}\left(0, T ; \mathbb{L}^{4}(\Omega)\right) \forall T>0$, we obtain the same regularity than for other boundary conditions cases, although the regularity in time is deduced only for each $T>0$ (and not for $T=+\infty$ yet).

\subsection{Application of maximum principle}

The proof of Theorem 1.9 is also true in the case of considering the time-dependent boundary condition for $Q$ (1.10). Therefore, using that $(\boldsymbol{u}, Q)$ is a weak solution with finite time regularity, we can deduce the uniform estimate

$$
Q \in L^{\infty}\left(0,+\infty ; \mathbb{L}^{\infty}(\Omega)\right)
$$

\subsection{Global in time estimates.}

An alternative treatment of the term $\left(\partial_{t} \widetilde{Q}, f(Q)\right)$ made in Subsection 4.1 can be achieved using the maximum principle given in Theorem 1.9. Indeed, since $|Q| \leq \alpha$ a.e. in $\Omega \times(0, T)$, then $|f(Q)| \leq \kappa(\alpha)$ a.e. in $\Omega \times(0, T)$ and:

$$
\left(\partial_{t} \widetilde{Q}, f(Q)\right) \leq \kappa(\alpha)\left\|\partial_{t} \widetilde{Q}\right\|_{\mathbb{L}^{1}(\Omega)}
$$

In this case, from (4.36) we arrive at:

$$
\begin{aligned}
\frac{d}{d t}\left(\frac{1}{2}\|\boldsymbol{u}\|_{\mathbf{L}^{2}(\Omega)}^{2}+\int_{\Omega} \widehat{\mathcal{E}}(Q) d \boldsymbol{x}\right) & +\nu\|\nabla \boldsymbol{u}\|_{\mathbf{L}^{2}(\Omega)}^{2}+\frac{\gamma}{2}\|-\varepsilon \Delta \widehat{Q}+f(Q)\|_{\mathbb{L}^{2}(\Omega)}^{2} \\
& \leq \frac{1}{2 \gamma}\left\|\partial_{t} \widetilde{Q}\right\|_{\mathbb{L}^{2}(\Omega)}^{2}+\kappa(\alpha)\left\|\partial_{t} \widetilde{Q}\right\|_{\mathbb{L}^{1}(\Omega)} .
\end{aligned}
$$

Assuming $\partial_{t} \widetilde{Q} \in L^{1}\left(0,+\infty ; \mathbb{L}^{1}(\Omega)\right) \cap L^{2}\left(0,+\infty ; \mathbb{L}^{2}(\Omega)\right)$, then the regularity at infinite time for $(\boldsymbol{u}, Q)$ given in $(1.11)$ is still preserved, hence the weak existence at infinite time with timedependent Dirichlet data for $Q$ holds.

\section{Uniqueness criteria of weak solutions (proof of Theorem 1.7)}

We decompose the term $H(Q)$ in the following way:

$$
H=-\varepsilon \Delta Q+F_{c}^{\prime}(Q)+F_{e}^{\prime}(Q):=H_{c}+F_{e}^{\prime}(Q)
$$


with $F_{c}(Q)=\frac{c}{4}|Q|^{4}$ a convex part of $F(Q)$ and $F_{e}(Q)=\frac{a}{2}|Q|^{2}-\frac{b}{3}\left(Q^{2}: Q\right)$ the rest one.

First of all, we study some properties of $F_{c}$ and $F_{e}$ using the expression of their first and second derivatives.

\subsection{About the function $F(Q)$}

Let $Q_{1}, Q_{2} \in \mathbb{R}^{3 \times 3}$ and $E=Q_{1}-Q_{2}$. Observe that:

$$
\frac{\partial F\left(Q_{1}\right)}{\partial Q_{m n}}-\frac{\partial F\left(Q_{2}\right)}{\partial Q_{m n}}=\frac{\partial^{2} F(R)}{\partial Q_{m n} \partial Q_{p q}} E_{p q},
$$

where $R=\xi Q_{1}+(1-\xi) Q_{2}$ with $\xi \in(0,1)$. Multiplying (5.39) by $E_{m n}$ :

$$
\left(\frac{\partial F\left(Q_{1}\right)}{\partial Q_{m n}}-\frac{\partial F\left(Q_{2}\right)}{\partial Q_{m n}}\right) E_{m n}=E_{m n} \frac{\partial^{2} F(R)}{\partial Q_{m n} \partial Q_{p q}} E_{p q}
$$

The first and second derivatives of $F(Q)$ are given by:

$$
\left\{\begin{array}{l}
\frac{\partial F_{e}(Q)}{\partial Q_{m n}}=a Q_{m n}-\frac{b}{3}\left(Q_{m i} Q_{i n}+Q_{m i} Q_{n i}+Q_{i m} Q_{i n}\right) \\
\frac{\partial F_{c}(Q)}{\partial Q_{m n}}=c|Q|^{2} Q_{m n}
\end{array}\right.
$$

and

$$
\left\{\begin{array}{l}
\frac{\partial^{2} F_{e}(Q)}{\partial Q_{m n} \partial Q_{p q}}=a \delta_{m p} \delta_{n q} \\
-\frac{b}{3}\left(Q_{q n} \delta_{m p}+Q_{m p} \delta_{n q}+Q_{n q} \delta_{m p}+Q_{m q} \delta_{n p}+Q_{p n} \delta_{m q}+Q_{p m} \delta_{n q}\right) \\
\frac{\partial^{2} F_{c}(Q)}{\partial Q_{m n} \partial Q_{p q}}=2 c Q_{p q} Q_{m n}+c|Q|^{2} \delta_{m p} \delta_{n q}
\end{array}\right.
$$

(recall that the Einstein summation convention over repeated indices is being used). Multiplying the second derivative of the convex part $F_{c}$ by $E_{p q}$ :

$$
\frac{\partial^{2} F_{c}(R)}{\partial Q_{m n} \partial Q_{p q}} E_{p q}=2 c(R: E) R_{m n}+c|R|^{2} E_{m n} .
$$

Then:

$$
\int_{\Omega} E_{m n} \frac{\partial^{2} F_{c}(Q)}{\partial Q_{m n} \partial Q_{p q}} E_{p q} d \boldsymbol{x}=c \int_{\Omega}\left[2(R: E)^{2}+|R|^{2}|E|^{2}\right] d \boldsymbol{x} \geq 0 .
$$

Moreover, using (5.39) and (5.41), we bound:

$$
\left\|\nabla F_{c}\left(Q_{1}\right)-\nabla F_{c}\left(Q_{2}\right)\right\|_{\mathbb{L}^{2}(\Omega)}^{2} \leq 3 c \int_{\Omega}|R|^{4}|E|^{2} d \boldsymbol{x} \leq 3 c\|R\|_{\mathbb{H}^{1}(\Omega)}^{4}\|E\|_{\mathbb{H}^{1}(\Omega)}^{2}
$$

From (5.40), we can deduce the following expression using the second derivative of the nonconvex part of $F(Q)$ :

$$
\frac{\partial^{2} F_{e}(R)}{\partial Q_{m n} \partial Q_{p q}} E_{p q}=a E_{m n}-\frac{b}{3}\left(E R+R E+E R^{t}+R E^{t}+E^{t} R+R^{t} E\right)_{m n}
$$


that together with (5.39) implies that:

$$
\left\|\nabla F_{e}\left(Q_{1}\right)-\nabla F_{e}\left(Q_{2}\right)\right\|_{\mathbb{L}^{2}(\Omega)}^{2} \leq C(a, b)\left(\|E\|_{\mathbb{L}^{2}(\Omega)}^{2}+\|R\|_{\mathbb{L}^{3}(\Omega)}^{2}\|E\|_{\mathbb{H}^{1}(\Omega)}^{2}\right) .
$$

\subsection{Proof of Theorem 1.7}

Let $\left(\boldsymbol{u}_{1}, q_{1}, Q_{1}, H^{1}\right),\left(\boldsymbol{u}_{2}, q_{2}, Q_{2}, H^{2}\right)$ be two solutions of problem (1.2)-(1.3) and set

$$
\boldsymbol{u}=\boldsymbol{u}_{1}-\boldsymbol{u}_{2}, \quad q=q_{1}-q_{2}, \quad E=Q_{1}-Q_{2}, \quad H_{c}=H_{c}^{1}-H_{c}^{2}
$$

(recall that $\left.\left(H_{c}\right)^{i}=-\varepsilon \Delta Q_{i}+F_{c}^{\prime}\left(Q_{i}\right)\right)$. Using decomposition for $H$ given in (5.38), taking into account that $H=H_{1}-H_{2}$ can be decomposed as

$$
\begin{aligned}
H & =-\varepsilon \Delta E+\nabla F_{c}\left(Q_{1}\right)-\nabla F_{c}\left(Q_{2}\right)+\nabla F_{e}\left(Q_{1}\right)-\nabla F_{e}\left(Q_{2}\right) \\
& =H_{c}+\nabla F_{e}\left(Q_{1}\right)-\nabla F_{e}\left(Q_{2}\right),
\end{aligned}
$$

and (2.22), then $\left(\boldsymbol{u}, q, E, H_{c}\right)$ satisfies in $\Omega \times(0, T)$ :

$$
\left\{\begin{aligned}
\partial_{t} \boldsymbol{u} & -\nu \Delta \boldsymbol{u}+\nabla q+\boldsymbol{u}_{1} \cdot \nabla \boldsymbol{u}-\left(H_{c}: \partial_{i} Q_{1}\right)_{i}-\nabla \cdot \sigma\left(H_{c}, Q_{1}\right) \\
& =-\boldsymbol{u} \cdot \nabla \boldsymbol{u}_{2}+\left(\nabla F_{e}\left(Q_{1}\right)-\nabla F_{e}\left(Q_{2}\right): \partial_{i} Q_{1}\right)_{i}+\left(H_{2}: \partial_{i} E\right)_{i} \\
& +\nabla \cdot \sigma\left(\nabla F_{e}\left(Q_{1}\right)-\nabla F_{e}\left(Q_{2}\right), Q_{1}\right)+\nabla \cdot \sigma\left(H_{2}, E\right) \\
\nabla \cdot \boldsymbol{u} & =0 \\
\partial_{t} E & +\gamma H_{c}+(\boldsymbol{u} \cdot \nabla) Q_{1}-S\left(\nabla \boldsymbol{u}, Q_{1}\right) \\
& =-\left(\boldsymbol{u}_{2} \cdot \nabla\right) E-\gamma\left(\nabla F_{e}\left(Q_{1}\right)-\nabla F_{e}\left(Q_{2}\right)\right)+S\left(\nabla \boldsymbol{u}_{2}, E\right) \\
-\varepsilon \Delta E & +\nabla F_{c}\left(Q_{1}\right)-\nabla F_{c}\left(Q_{2}\right)-H_{c}=0
\end{aligned}\right.
$$

Taking $(\boldsymbol{u}, q)$ as test function in $(5.45)_{1-2}, H_{c}$ as test function in $(5.45)_{3}$ and $\partial_{t} E$ as test function in $(5.45)_{4}$, the following terms cancel (as in the deduction of the energy law):

$$
\begin{gathered}
\left(\boldsymbol{u}_{1} \cdot \nabla \boldsymbol{u}, \boldsymbol{u}\right)=0, \\
-\left(H_{c}: \partial_{i} Q_{1}, \boldsymbol{u}_{i}\right)+\left(\boldsymbol{u} \cdot \nabla Q_{1}, H_{c}\right)=0, \\
\left(\sigma\left(H_{c}, Q_{1}\right), \nabla \boldsymbol{u}\right)-\left(S\left(\nabla \boldsymbol{u}, Q_{1}\right), H_{c}\right)=0 .
\end{gathered}
$$

Now, we analyze the remaining terms. First, we focus on the effect of the test functions over the LHS of each equation of (5.45):

$$
\left(\partial_{t} \boldsymbol{u}-\nu \Delta \boldsymbol{u}+\nabla q+\boldsymbol{u}_{1} \cdot \nabla \boldsymbol{u}, \boldsymbol{u}\right)=\frac{1}{2} \frac{d}{d t}\|\boldsymbol{u}\|_{\mathbf{L}^{2}(\Omega)}^{2}+\nu\|\nabla \boldsymbol{u}\|_{\mathbf{L}^{2}(\Omega)}^{2}
$$




$$
\begin{aligned}
\gamma\left(H_{c}, H_{c}\right)=\gamma\left\|H_{c}\right\|_{\mathbb{L}^{2}(\Omega)}^{2} \\
\left(\partial_{t} E, H_{c}\right)=\left(E_{t},-\varepsilon \Delta E+\nabla F_{c}\left(Q_{1}\right)-\nabla F_{c}\left(Q_{2}\right)\right) \\
=\frac{\varepsilon}{2} \frac{d}{d t}\|\nabla E\|_{\mathbb{L}^{2}(\Omega)}^{2}+\left(\partial_{t} E, \nabla F_{c}\left(Q_{1}\right)-\nabla F_{c}\left(Q_{2}\right)\right) \\
=\frac{\varepsilon}{2} \frac{d}{d t}\|\nabla E\|_{\mathbb{L}^{2}(\Omega)}^{2}+\partial_{t} E_{m n} \frac{\partial^{2} F_{c}(R)}{\partial Q_{m n} \partial Q_{p q}} E_{p q},
\end{aligned}
$$

for some $R=\xi Q_{1}+(1-\xi) Q_{2}$ with $\xi \in(0,1)$. Since:

$$
\begin{aligned}
\frac{1}{2} \frac{d}{d t}\left[E_{m n} \frac{\partial^{2} F_{c}(R)}{\partial Q_{m n} \partial Q_{p q}} E_{p q}\right] & =\partial_{t} E_{m n} \frac{\partial^{2} F_{c}(R)}{\partial Q_{m n} \partial Q_{p q}} E_{p q} \\
& +\frac{1}{2} E_{m n} \frac{\partial^{3} F_{c}(R)}{\partial Q_{m n} \partial Q_{p q} \partial Q_{r s}}\left(\partial_{t} R_{r s}\right) E_{p q}
\end{aligned}
$$

then, from (5.47),

$$
\begin{aligned}
\left(\partial_{t} E, H_{c}\right) & =\frac{1}{2} \frac{d}{d t}\left(\varepsilon\|\nabla E\|_{\mathbb{L}^{2}(\Omega)}^{2}+\int_{\Omega} E_{m n} \frac{\partial^{2} F_{c}(R)}{\partial Q_{m n} \partial Q_{p q}} E_{p q} d \boldsymbol{x}\right) \\
& -\frac{1}{2} \int_{\Omega} E_{m n} \frac{\partial^{3} F_{c}(R)}{\partial Q_{m n} \partial Q_{p q} \partial Q_{r s}}\left(\partial_{t} R_{r s}\right) E_{p q} d \boldsymbol{x} .
\end{aligned}
$$

Collecting the previous computations, we arrive at the following inequality:

$$
\begin{aligned}
\frac{1}{2} \frac{d}{d t} & \left(\|\boldsymbol{u}\|_{\mathbf{L}^{2}(\Omega)}^{2}+\varepsilon\|\nabla E\|_{\mathbb{L}^{2}(\Omega)}^{2}+\int_{\Omega} E_{m n} \frac{\partial^{2} F_{c}(R)}{\partial Q_{m n} \partial Q_{p q}} E_{p q} d \boldsymbol{x}\right) \\
& +\nu\|\nabla \boldsymbol{u}\|_{\mathbf{L}^{2}(\Omega)}^{2}+\gamma\left\|H_{c}\right\|_{\mathbb{L}^{2}(\Omega)}^{2} \\
& \leq-\int_{\Omega} \boldsymbol{u} \cdot \nabla \boldsymbol{u}_{2} \cdot \boldsymbol{u} d \boldsymbol{x}+\int_{\Omega}\left[(\boldsymbol{u} \cdot \nabla) Q_{1}\right]:\left(\nabla F_{e}\left(Q_{1}\right)-\nabla F_{e}\left(Q_{2}\right)\right) d \boldsymbol{x} \\
& +\int_{\Omega}[(\boldsymbol{u} \cdot \nabla) E]: H_{2} d \boldsymbol{x}-\int_{\Omega} \sigma\left(\nabla F_{e}\left(Q_{1}\right)-\nabla F_{e}\left(Q_{2}\right), Q_{1}\right): \nabla \boldsymbol{u} d \boldsymbol{x} \\
& -\int_{\Omega} \sigma\left(H_{2}, E\right): \nabla \boldsymbol{u} d \boldsymbol{x}-\int_{\Omega}\left[\left(\boldsymbol{u}_{2} \cdot \nabla\right) E\right]: H_{c} d \boldsymbol{x} \\
& -\gamma \int_{\Omega}\left(\nabla F_{e}\left(Q_{1}\right)-\nabla F_{e}\left(Q_{2}\right)\right): H_{c} d \boldsymbol{x}+\int_{\Omega} S\left(\nabla \boldsymbol{u}_{2}, E\right): H_{c} d \boldsymbol{x} \\
& +\frac{1}{2} \int_{\Omega} E_{m n} \frac{\partial^{3} F_{c}(R)}{\partial Q_{m n} \partial Q_{p q} \partial Q_{r s}}\left(\partial_{t} R_{r s}\right) E_{p q} d \boldsymbol{x}:=\sum_{i=1}^{9} I_{i} .
\end{aligned}
$$

In a first attempt, we can bound the Navier-Stokes nonlinearity using the Berselli's regularity 
criteria as follows:

$$
\begin{aligned}
\left|I_{1}\right| & \leq\|\boldsymbol{u}\|_{\mathbf{L}^{2 q^{\prime}}(\Omega)}^{2}\left\|\nabla \boldsymbol{u}_{2}\right\|_{\mathbf{L}^{q}(\Omega)} \leq C\|\boldsymbol{u}\|_{\mathbf{L}^{2}(\Omega)}^{\frac{2 q-3}{q}}\|\nabla \boldsymbol{u}\|_{\mathbf{L}^{2}(\Omega)}^{\frac{3}{q}}\left\|\nabla \boldsymbol{u}_{2}\right\|_{\mathbf{L}^{q}(\Omega)} \\
& \leq \frac{\nu}{10}\|\nabla \boldsymbol{u}\|_{\mathbf{L}^{2}(\Omega)}^{2}+C_{\nu}\left\|\nabla \boldsymbol{u}_{2}\right\|_{\mathbf{L}^{q}(\Omega)}^{\frac{2 q}{2 q-3}}\|\boldsymbol{u}\|_{\mathbf{L}^{2}(\Omega)}^{2} .
\end{aligned}
$$

By using (5.44) we can bound $I_{2}$ as:

$$
\begin{aligned}
\left|I_{2}\right| & \leq\|\boldsymbol{u}\|_{\mathbf{L}^{6}(\Omega)}\left\|\nabla Q_{1}\right\|_{\mathbb{L}^{3}(\Omega)}\left\|\nabla F_{e}\left(Q_{1}\right)-\nabla F_{e}\left(Q_{2}\right)\right\|_{\mathbb{L}^{2}(\Omega)} \\
& \leq \frac{\nu}{10}\|\nabla \boldsymbol{u}\|_{\mathbf{L}^{2}(\Omega)}^{2}+C_{\nu}\left\|\nabla Q_{1}\right\|_{\mathbb{L}^{3}(\Omega)}^{2}\left(\|E\|_{\mathbb{L}^{2}(\Omega)}^{2}+\|R\|_{\mathbb{L}^{3}(\Omega)}^{2}\|E\|_{\mathbb{H}^{1}(\Omega)}^{2}\right) .
\end{aligned}
$$

Taking into account that:

$$
\begin{aligned}
\|E\|_{\mathbb{H}^{2}(\Omega)} & \leq\|\Delta E\|_{\mathbb{L}^{2}(\Omega)}+\|E\|_{\mathbb{L}^{2}(\Omega)} \\
& \leq\left\|H_{c}\right\|_{\mathbb{L}^{2}(\Omega)}+\left\|\nabla F_{c}\left(Q_{1}\right)-\nabla F_{c}\left(Q_{2}\right)\right\|_{\mathbb{L}^{2}(\Omega)}+\|E\|_{\mathbb{L}^{2}(\Omega)},
\end{aligned}
$$

we can bound the $I_{3}$ and $I_{4}$-terms as follows:

$$
\begin{aligned}
\left|I_{3}\right| & \leq\|\boldsymbol{u}\|_{\mathbf{L}^{\frac{2 s}{s-1}(\Omega)}}\|\nabla E\|_{\mathbb{L}^{\frac{2 s}{s-1}(\Omega)}}\left\|H_{2}\right\|_{\mathbb{L}^{s}(\Omega)} \\
& \leq\|\boldsymbol{u}\|_{\mathbf{L}^{2}(\Omega)}^{\frac{2 s-3}{2 s}}\|\nabla \boldsymbol{u}\|_{\mathbf{L}^{2}(\Omega)}^{\frac{3}{2 s}}\|\nabla E\|_{\mathbf{L}^{2}(\Omega)}^{\frac{2 s-3}{2 s}}\|E\|_{\mathbb{H}^{2}(\Omega)}^{\frac{3}{2 s}}\left\|H_{2}\right\|_{\mathbb{L}^{s}(\Omega)} \\
& \leq \frac{\nu}{10}\|\nabla \boldsymbol{u}\|_{\mathbf{L}^{2}(\Omega)}^{2}+\frac{\gamma}{12}\left\|H_{c^{\prime}}\right\|_{\mathbb{L}^{2}(\Omega)}^{2} \\
& +C_{\nu, \varepsilon}\left\{\left\|H_{2}\right\|_{\mathbb{L}^{s}(\Omega)}^{\frac{2 s}{2 s-3}}\left(\|\boldsymbol{u}\|_{\mathbf{L}^{2}(\Omega)}^{2}+\|E\|_{\mathbb{H}^{1}(\Omega)}^{2}\right)\right. \\
& \left.+\|\mathbb{R}\|_{\mathbb{H}^{1}(\Omega)}^{4}\|E\|_{\mathbb{H}^{1}(\Omega)}^{2}+\|E\|_{\mathbb{L}^{2}(\Omega)}^{2}\right\}
\end{aligned}
$$

and

$$
\begin{aligned}
& \left|I_{4}\right| \leq C\left\|Q_{1}\right\|_{\mathbb{L}^{\infty}(\Omega)}\left\|\nabla F_{e}\left(Q_{1}\right)-\nabla F_{e}\left(Q_{2}\right)\right\|_{\mathbb{L}^{2}(\Omega)}\|\nabla \boldsymbol{u}\|_{\mathbf{L}^{2}(\Omega)} \\
& \quad \leq \frac{\nu}{10}\|\nabla \boldsymbol{u}\|_{\mathbf{L}^{2}(\Omega)}^{2}+C_{\nu}\left\|Q_{1}\right\|_{\mathbb{H}^{1}(\Omega)}\left\|Q_{1}\right\|_{\mathbb{H}^{2}(\Omega)}\left(\|E\|_{\mathbb{L}^{2}(\Omega)}^{2}+\|R\|_{\mathbb{L}^{3}(\Omega)}^{2}\|E\|_{\mathbb{H}^{1}(\Omega)}^{2}\right) .
\end{aligned}
$$

By using again (5.50), we can bound (for $2 \leq s \leq 3$ ):

$$
\begin{aligned}
\left|I_{5}\right| & \leq\|E\|_{\mathbb{L}^{\frac{2 s}{s-2}(\Omega)}}\left\|H_{2}\right\|_{\mathbb{L}^{s}(\Omega)}\|\nabla \boldsymbol{u}\|_{\mathbf{L}^{2}(\Omega)} \\
& \leq C\|E\|_{\mathbb{W}^{1, \frac{6 s}{5 s-6}(\Omega)}}\left\|H_{2}\right\|_{\mathbb{L}^{s}(\Omega)}\|\nabla \boldsymbol{u}\|_{\mathbf{L}^{2}(\Omega)} \\
& \leq C\|E\|_{\mathbb{H}^{1}(\Omega)}^{\frac{2 s-3}{s}}\|E\|_{\mathbb{H}^{2}(\Omega)}^{\frac{3-s}{s}}\left\|H_{2}\right\|_{\mathbb{L}^{s}(\Omega)}\|\nabla \boldsymbol{u}\|_{\mathbf{L}^{2}(\Omega)} \\
& \leq \frac{\nu}{10}\|\nabla \boldsymbol{u}\|_{\mathbf{L}^{2}(\Omega)}^{2}+\frac{\gamma}{12}\left\|H_{C}\right\|_{\mathbb{L}^{2}(\Omega)}^{2} \\
& +C_{\nu, \varepsilon}\left(\left\|H_{2}\right\|_{\mathbb{L}^{s}(\Omega)}^{\frac{2 s}{2 s-3}}+\|R\|_{\mathbb{H}^{1}(\Omega)}^{4}+1\right)\|E\|_{\mathbb{H}^{1}(\Omega)}^{2} .
\end{aligned}
$$


In order to bound the $I_{6}$-term, we decompose as:

$$
I_{6}=\varepsilon \int_{\Omega}\left(\boldsymbol{u}_{2} \cdot \nabla\right) E: \Delta E d \boldsymbol{x}-\int_{\Omega}\left(\boldsymbol{u}_{2} \cdot \nabla\right) E:\left(\nabla F_{c}\left(Q_{1}\right)-\nabla F_{c}\left(Q_{2}\right)\right) d \boldsymbol{x}:=J_{1}+J_{2} .
$$

Integrating by parts the $J_{1}$-term,

$$
J_{1}=\varepsilon \int_{\Omega}\left|\nabla \boldsymbol{u}_{2}\right||\nabla E|^{2} d \boldsymbol{x}
$$

hence, we can bound it like the $I_{1}$-term,

$$
\begin{aligned}
\left|J_{1}\right| & \leq C\left\|\nabla \boldsymbol{u}_{2}\right\|_{\mathbf{L}^{q}(\Omega)}\|\nabla E\|_{\mathbb{L}^{2 q^{\prime}}(\Omega)}^{2} \leq C\left\|\nabla \boldsymbol{u}_{2}\right\|_{\mathbf{L}^{q}(\Omega)}\|E\|_{\mathbb{H}^{1}(\Omega)}^{\frac{2 q-3}{q}}\|E\|_{\mathbb{H}^{2}(\Omega)}^{\frac{3}{q}} \\
& \leq C\left\|\nabla \boldsymbol{u}_{2}\right\|_{\mathbf{L}^{q}(\Omega)}\|E\|_{\mathbb{H}^{1}(\Omega)}^{\frac{2 q-3}{q}} \\
& \times\left(\left\|H_{c}\right\|_{\mathbb{L}^{2}(\Omega)}+\left\|\nabla F_{c}\left(Q_{1}\right)-\nabla F_{c}\left(Q_{2}\right)\right\|_{\mathbb{L}^{2}(\Omega)}+\|E\|_{\mathbb{L}^{2}(\Omega)}\right)^{\frac{3}{q}} \\
& \leq \frac{\gamma}{12}\left\|H_{c}\right\|_{\mathbb{L}^{2}(\Omega)}^{2} \\
& +C_{\varepsilon}\left\{\|E\|_{\mathbb{L}^{2}(\Omega)}^{2}+\left(\|R\|_{\mathbb{H}^{1}(\Omega)}^{4}+\left\|\nabla \boldsymbol{u}_{2}\right\|_{\mathbf{L}^{q}(\Omega)}^{\frac{2 q}{2 q-3}}\right)\|E\|_{\mathbb{H}^{1}(\Omega)}^{2}\right\}
\end{aligned}
$$

and using (5.43),

$$
\begin{aligned}
\left|J_{2}\right| & \leq\left\|\boldsymbol{u}_{2}\right\|_{\mathbf{L}^{3}(\Omega)}\|\nabla E\|_{\mathbb{L}^{6}(\Omega)}\left\|\nabla F_{c}\left(Q_{1}\right)-\nabla F_{c}\left(Q_{2}\right)\right\|_{\mathbb{L}^{2}(\Omega)} \\
& \leq\left\|\boldsymbol{u}_{2}\right\|_{\mathbf{L}^{3}(\Omega)}\left(\left\|H_{c}\right\|_{\mathbb{L}^{2}(\Omega)}+\left\|\nabla F_{c}\left(Q_{1}\right)-\nabla F_{c}\left(Q_{2}\right)\right\|_{\mathbb{L}^{2}(\Omega)}+\|E\|_{\mathbb{L}^{2}(\Omega)}\right) \\
& \times\left\|\nabla F_{c}\left(Q_{1}\right)-\nabla F_{c}\left(Q_{2}\right)\right\|_{\mathbb{L}^{2}(\Omega)} \\
& \leq \frac{\gamma}{12}\left\|H_{c}\right\|_{\mathbb{L}^{2}(\Omega)}^{2}+C_{\varepsilon}\left\{\left(\left\|\boldsymbol{u}_{2}\right\|_{\mathbf{L}^{3}(\Omega)}^{2}+1\right)\|R\|_{\mathbb{H}^{1}(\Omega)}^{4}\|E\|_{\mathbb{H}^{1}(\Omega)}^{2}+\|E\|_{\mathbb{L}^{2}(\Omega)}^{2}\right\} .
\end{aligned}
$$

Now, we bound the $I_{7}$ and $I_{8}$-terms as follows:

$$
\begin{aligned}
&\left|I_{7}\right| \leq \gamma\left\|\nabla F_{e}\left(Q_{1}\right)-\nabla F_{e}\left(Q_{2}\right)\right\|_{\mathbb{L}^{2}(\Omega)}\left\|H_{c}\right\|_{\mathbb{L}^{2}(\Omega)} \\
& \leq \frac{\gamma}{12}\left\|H_{c}\right\|_{\mathbb{L}^{2}(\Omega)}^{2}+C_{\varepsilon, a, b}\left(\|E\|_{\mathbb{L}^{2}(\Omega)}^{2}+\|R\|_{\mathbb{L}^{3}(\Omega)}^{2}\|E\|_{\mathbb{H}^{1}(\Omega)}^{2}\right) \\
&\left|I_{8}\right| \leq\left\|\nabla \boldsymbol{u}_{2}\right\|_{\mathbf{L}^{q}(\Omega)}\|E\|_{\mathbb{L}^{\frac{2 q}{q-2}(\Omega)}}\left\|H_{c}\right\|_{\mathbb{L}^{2}(\Omega)} \\
& \leq C\left\|\nabla \boldsymbol{u}_{2}\right\|_{\mathbf{L}^{q}(\Omega)}\|E\|_{\mathbb{W}^{1, \frac{6 q}{5 q-6}(\Omega)}}\left\|H_{c}\right\|_{\mathbb{L}^{2}(\Omega)} \\
& \leq C\left\|\nabla \boldsymbol{u}_{2}\right\|_{\mathbf{L}^{q}(\Omega)}\|E\|_{\mathbb{H}^{1}(\Omega)}^{\frac{2 q-3}{q}}\|E\|_{\mathbb{H}^{2}(\Omega)}^{\frac{3-q}{q}}\left\|H_{c}\right\|_{\mathbb{L}^{2}(\Omega)} \\
& \leq \frac{\gamma}{12}\left\|H_{c}\right\|_{\mathbb{L}^{2}(\Omega)}^{2}+C_{\varepsilon}\left(\left\|\nabla \boldsymbol{u}_{2}\right\|_{\mathbf{L}^{q}(\Omega)}^{\frac{2 q}{2 q-3}}+\|R\|_{\mathbb{H}^{1}(\Omega)}^{4}+1\right)\|E\|_{\mathbb{H}^{1}(\Omega)}^{2} .
\end{aligned}
$$


where we have used (5.50) and (5.43). Note that in the previous estimate of the $I_{8}$-term the constraint $2 \leq q \leq 3$ must be imposed.

In order to bound the $I_{9}$-term, we take into account that:

$$
\frac{\partial^{3} F_{c}(R)}{\partial Q_{m n} \partial Q_{p q} \partial Q_{r s}}=2 c\left(R_{p q} \delta_{m r} \delta_{n s}+R_{m n} \delta_{p r} \delta_{q s}+R_{r s} \delta_{m p} \delta_{n q}\right),
$$

hence

$$
\begin{aligned}
\left|I_{9}\right| & =\left|c \int_{\Omega}\left(2(R: E)\left(\partial_{t} R: E\right)+\left(R: \partial_{t} R\right)|E|^{2}\right) d \boldsymbol{x}\right| \\
& \leq 3 c \int_{\Omega}|E|^{2}|R|\left|\partial_{t} R\right| d \boldsymbol{x} .
\end{aligned}
$$

In particular,

$$
\left|I_{9}\right| \leq\left\|\partial_{t} R\right\|_{\mathbb{L}^{2}(\Omega)}\|R\|_{\mathbb{L}^{6}(\Omega)}\|E\|_{\mathbb{L}^{6}(\Omega)}^{2} \leq C\left\|\partial_{t} R\right\|_{\mathbb{L}^{2}(\Omega)}\|R\|_{\mathbb{H}^{1}(\Omega)}\|E\|_{\mathbb{H}^{1}(\Omega)}^{2}
$$

Collecting all the previous estimates in (5.49) and using (5.42) we obtain the following inequality:

$$
\begin{gathered}
\frac{1}{2} \frac{d}{d t}\left(\|\boldsymbol{u}\|_{\mathbf{L}^{2}(\Omega)}^{2}+\varepsilon\|\nabla E\|_{\mathbb{L}^{2}(\Omega)}^{2}+c \int_{\Omega}\left[2(R: E)^{2}+|R|^{2}|E|^{2}\right] d \boldsymbol{x}\right) \\
\quad+\nu\|\nabla \boldsymbol{u}\|_{\mathbf{L}^{2}(\Omega)}^{2}+\gamma\left\|H_{C}\right\|_{\mathbb{L}^{2}(\Omega)}^{2} \leq C(t)\left(\|\boldsymbol{u}\|_{\mathbf{L}^{2}(\Omega)}^{2}+\|E\|_{\mathbb{H}^{1}(\Omega)}^{2}\right)
\end{gathered}
$$

where

$$
\begin{aligned}
C(t) & =C\left(\left\|\nabla \boldsymbol{u}_{2}\right\|_{\mathbf{L}^{q}(\Omega)}^{\frac{2 q}{2 q-3}}+\left\|H_{2}\right\|_{\mathbb{L}^{s}(\Omega)}^{\frac{2 s}{2 s-3}}+\left(\left\|\boldsymbol{u}_{2}\right\|_{\mathbf{L}^{3}(\Omega)}^{2}+1\right)\|R\|_{\mathbb{H}^{1}(\Omega)}^{4}\right. \\
& \left.+\|R\|_{\mathbb{L}^{3}(\Omega)}^{2}\left(1+\left\|Q_{1}\right\|_{\mathbb{H}^{1}(\Omega)}\left\|Q_{1}\right\|_{\mathbb{H}^{2}(\Omega)}\right)+\|R\|_{\mathbb{H}^{1}(\Omega)}\left\|\partial_{t} R\right\|_{\mathbb{L}^{2}(\Omega)}\right) .
\end{aligned}
$$

By using the weak regularity of $(\boldsymbol{u}, Q)$ given in (1.11), we need to impose the additional hypothesis given in (1.15) to guarantee that $C(t) \in L^{1}(0, T)$ (recall that the weak regularity implies $\left.\partial_{t} R \in L^{4 / 3}\left(0, T ; \mathbb{L}^{2}(\Omega)\right)\right)$. Taking into account that:

$$
H_{2}=-\varepsilon \Delta Q_{2}+f\left(Q_{2}\right)
$$

the fact that $Q_{2} \in L^{\infty}\left(0, T ; \mathbb{H}^{1}(\Omega)\right) \cap L^{2}\left(0, T ; \mathbb{H}^{2}(\Omega)\right)$ implies that $f\left(Q_{2}\right) \in L^{\frac{2 s}{2 s-3}}\left(0, T ; \mathbb{L}^{s}(\Omega)\right)$ $(s>1)$ because of (focusing on the less regular term):

$$
\begin{aligned}
\left\||Q|^{2} Q\right\|_{\mathbb{L}^{s}(\Omega)} & =\|Q\|_{\mathbb{L}^{3 s}(\Omega)}^{3} \\
& \leq \begin{cases}C\|Q\|_{\mathbb{H}^{1}(\Omega)}^{3} & \text { for } s \in(1,2] \\
C\|Q\|_{\mathbb{W}^{1, \frac{3 s}{s+1}(\Omega)}}^{3} \leq C\|Q\|_{\mathbb{H}^{1}(\Omega)}^{\frac{3(s+2)}{2 s}}\|Q\|_{\mathbb{H}^{2}(\Omega)}^{\frac{3(s-2)}{2 s}} & \text { for } s>2\end{cases}
\end{aligned}
$$


hence $|Q|^{2} Q \in L^{\frac{2 s}{2 s-3}}\left(0, T ; \mathbb{L}^{s}(\Omega)\right)$ iff $\frac{3(s-2)}{2 s-3} \leq 2$ which is always true. Therefore the additional regularity $H_{2} \in L^{\frac{2 s}{2 s-3}}\left(0, T ; \mathbb{L}^{s}(\Omega)\right)$ reduces to:

$$
\Delta Q_{2} \in L^{\frac{2 s}{2 s-3}}\left(0, T ; \mathbb{L}^{s}(\Omega)\right) .
$$

In order to obtain the $H^{1}(\Omega)$-norm for $E$ in (5.51), we change the decomposition of $F(Q)=$ $F_{c}(Q)+F_{e}(Q)$ by $F(Q)=\widetilde{F}_{c}(Q)+\widetilde{F}_{e}(Q)$, given by:

$$
\begin{aligned}
F(Q) & =\left[F_{c}(Q)+\frac{\varepsilon}{2}|Q|^{2}\right]+\left[F_{e}(Q)-\frac{\varepsilon}{2}|Q|^{2}\right] \\
& =\widetilde{F}_{c}(Q)+\widetilde{F}_{e}(Q) .
\end{aligned}
$$

Observe that the term related to $\nabla F_{c}$ in $(5.47)$ is replaced by:

$$
\left(\partial_{t} E, \nabla \widetilde{F}_{c}\left(Q_{1}\right)-\nabla \widetilde{F}_{c}\left(Q_{2}\right)\right)=\left(\partial_{t} E\right)_{m n} \frac{\partial^{2} F_{c}(R)}{\partial Q_{m n} \partial Q_{p q}} E_{p q}+\frac{\varepsilon}{2} \partial_{t}\left(|E|^{2}\right),
$$

where $R=\xi Q_{1}+(1-\xi) Q_{2}$ with $\xi \in(0,1)$, which implies that (5.48) is replaced by:

$$
\begin{aligned}
\left(\partial_{t} E, H_{c}\right) & =\frac{1}{2} \frac{d}{d t}\left(\varepsilon\|E\|_{\mathbb{H}^{1}(\Omega)}^{2}+\int_{\Omega} E_{m n} \frac{\partial^{2} F_{c}(R)}{\partial Q_{m n} \partial Q_{p q}} E_{p q} d \boldsymbol{x}\right) \\
& -\frac{1}{2} \int_{\Omega} E_{m n} \frac{\partial^{3} F_{c}(R)}{\partial Q_{m n} \partial Q_{p q} \partial Q_{r s}}\left(\partial_{t} R_{r s}\right) E_{p q} d \boldsymbol{x} .
\end{aligned}
$$

On the other hand, (5.43) is replaced by:

$$
\left\|\nabla \widetilde{F}_{c}\left(Q_{1}\right)-\nabla \widetilde{F}_{c}\left(Q_{2}\right)\right\|_{\mathbb{L}^{2}(\Omega)}^{2} \leq 3 c \int_{\Omega}\|R\|_{\mathbb{H}^{1}(\Omega)}^{4}\|E\|_{\mathbb{H}^{1}(\Omega)}^{2}+\varepsilon\|E\|_{\mathbb{L}^{2}(\Omega)}^{2}
$$

and (5.44) is replaced by:

$$
\left\|\nabla \widetilde{F}_{e}\left(Q_{1}\right)-\nabla \widetilde{F}_{e}\left(Q_{2}\right)\right\|_{\mathbb{L}^{2}(\Omega)}^{2} \leq C(a-\varepsilon, b)\left(\|E\|_{\mathbb{L}^{2}(\Omega)}^{2}+\|R\|_{\mathbb{L}^{3}(\Omega)}^{2}\|E\|_{\mathbb{H}^{1}(\Omega)}^{2}\right) .
$$

Therefore, (5.51) is replaced by:

$$
\begin{array}{r}
\frac{1}{2} \frac{d}{d t}\left(\|\boldsymbol{u}\|_{\mathbf{L}^{2}(\Omega)}^{2}+\varepsilon\|E\|_{\mathbb{H}^{1}(\Omega)}^{2}+c \int_{\Omega}\left[2(R: E)^{2}+|R|^{2}|E|^{2}\right] d \boldsymbol{x}\right) \\
\quad+\nu\|\nabla \boldsymbol{u}\|_{\mathbf{L}^{2}(\Omega)}^{2}+\gamma\left\|H_{c}\right\|_{\mathbb{L}^{2}(\Omega)}^{2} \leq C(t)\left(\|\boldsymbol{u}\|_{\mathbf{L}^{2}(\Omega)}^{2}+\|E\|_{\mathbb{H}^{1}(\Omega)}^{2}\right)
\end{array}
$$

where $C(t)$ is given in (5.52). Then, the proof of Theorem 1.7 is easily deduced.

Remark 5.1 For the Nematic Liquid Crystal model studied in [12] (where the role of $Q$ is given by $\boldsymbol{d})$ without stretching terms, the uniqueness criteria (1.15) imposed in Theorem 1.7 is also a regularity criteria, which suffices to obtain global in time strong regularity (essentially obtaining one higher degree of regularity for $(\boldsymbol{u}, Q)$ than weak regularity given in (1.11)). But, for systems with stretching terms (see for instance [18]) this type of regularity results is not clear, excepting the case of space-periodic boundary conditions. 


\section{A maximum principle (Proof of Theorem 1.9)}

Considering the inner product of the $Q$-system by $Q$ and taking into account that the stretching terms vanish (using Lemma 3.1), that is,

$$
S(\nabla \boldsymbol{u}, Q): Q=\left(\nabla \boldsymbol{u} Q^{t}-Q^{t} \nabla \boldsymbol{u}\right): Q=\nabla \boldsymbol{u}:(Q Q)-\nabla \boldsymbol{u}:(Q Q)=0
$$

we obtain:

$$
\begin{aligned}
\frac{1}{2} \partial_{t}\left(|Q|^{2}\right) & +\boldsymbol{u} \cdot \nabla\left(\frac{|Q|^{2}}{2}\right)-\gamma \frac{\varepsilon}{2} \Delta\left(|Q|^{2}\right) \\
& +\gamma \varepsilon|\nabla Q|^{2}+\gamma f(Q): Q=0, \quad \text { in } \Omega \times(0,+\infty) .
\end{aligned}
$$

In the search of a value $\alpha>0$ to bound point-wisely $|Q(\boldsymbol{x}, t)|$, we observe from (6.54) that:

$$
\begin{aligned}
\partial_{t}\left(|Q|^{2}-\alpha^{2}\right) & +\boldsymbol{u} \cdot \nabla\left(|Q|^{2}-\alpha^{2}\right)-\gamma \varepsilon \Delta\left(|Q|^{2}-\alpha^{2}\right) \\
& +2 \gamma f(Q): Q \leq 0, \quad \text { in } \Omega \times(0,+\infty) .
\end{aligned}
$$

Testing $(6.55)$ by $\psi(Q):=\left(|Q|^{2}-\alpha^{2}\right)_{+}$and integrating in $\Omega$, we obtain:

$$
\begin{aligned}
& \frac{d}{d t}\|\psi(Q)\|_{L^{2}(\Omega)}^{2}+\gamma \varepsilon\|\nabla \psi(Q)\|_{L^{2}(\Omega)}^{2}-\gamma \varepsilon \int_{\Gamma} \partial_{\boldsymbol{n}} \psi(Q) \psi(Q) d \sigma \\
& \quad+2 \gamma \int_{\Omega}(f(Q): Q) \psi(Q) d \boldsymbol{x} \leq 0 .
\end{aligned}
$$

The boundary term $-\gamma \varepsilon \int_{\Gamma} \partial_{n} \psi(Q) \psi(Q) d \sigma$ vanishes. Indeed, in the case of Dirichlet boundary condition $\left.Q\right|_{\Gamma}=Q_{\Gamma}$ one has $\left.\psi(Q)\right|_{\Gamma}=0$ (because $\left|Q_{\Gamma}\right| \leq \alpha$ ) and in the case of Neumann boundary condition $\left.\partial_{n} Q\right|_{\Gamma}=0$ one has $\left.\partial_{n} \psi(Q)\right|_{\Gamma}=\mathbf{0}$. Therefore, in both cases the boundary integral term of (6.56) vanishes. On the other hand, from definition of $f(Q)$ :

$$
f(Q): Q=a|Q|^{2}-b\left(Q^{2}: Q\right)+c|Q|^{4}
$$

Using Young's inequality:

$$
b\left(Q^{2}: Q\right) \leq \frac{c}{2}|Q|^{4}+\frac{b^{2}}{2 c}|Q|^{2}
$$

one has:

$$
f(Q): Q \geq \frac{c}{2}|Q|^{4}+\left(a-\frac{b^{2}}{2 c}\right)|Q|^{2}=\frac{c}{2}|Q|^{2}\left(|Q|^{2}-\beta\right)
$$

with $\beta=\frac{b^{2}}{c^{2}}-\frac{2 a}{c}$. For instance, note that $\beta>0$ if $a<0$. Thus, considering $\alpha>0$ such that $\alpha^{2} \geq \beta$, we have:

$$
(f(Q): Q) \psi(Q) \geq \frac{c}{2}|Q|^{2}\left(|Q|^{2}-\beta\right)\left(|Q|^{2}-\alpha^{2}\right)_{+} \geq 0
$$


In this case, from (6.56) one deduces:

$$
\frac{d}{d t}\|\psi(Q)\|_{L^{2}(\Omega)}^{2}+\gamma \varepsilon\|\nabla \psi(Q)\|_{L^{2}(\Omega)}^{2} \leq 0
$$

hence

$$
\|\psi(Q(t))\|_{L^{2}(\Omega)}^{2} \leq\left\|\psi\left(Q_{0}\right)\right\|_{L^{2}(\Omega)}^{2}=\left\|\left(\left|Q_{0}\right|^{2}-\alpha^{2}\right)_{+}\right\|_{L^{2}(\Omega)}^{2}=0 .
$$

Therefore, the Maximum's Principle $\|Q(t)\|_{\mathbb{L}^{\infty}(\Omega)} \leq \alpha$ in $(0,+\infty)$ is deduced.

\section{How to enforce symmetry and traceless to $Q$}

\subsection{Rewriting $H$ to obtain traceless of $Q$}

In the search of a model satisfying $\operatorname{tr}(Q)=0$, we will use the modified expression for $H$ replacing (1.6) by (1.16) with the scalar function $\alpha(Q)$ is given in (1.17).

First of all, observe that $\operatorname{tr}(S(\nabla \boldsymbol{u}, Q))=0$ (using that $\operatorname{tr}(A B)=\operatorname{tr}(B A)), \operatorname{tr}\left(\partial_{t} Q\right)=$ $\partial_{t} \operatorname{tr}(Q), \operatorname{tr}(\boldsymbol{u} \cdot \nabla Q)=\boldsymbol{u} \cdot \nabla \operatorname{tr}(Q)$ and

$$
\operatorname{tr}(H)=-\varepsilon \Delta \operatorname{tr}(Q)+\left[a+c|Q|^{2}\right] \operatorname{tr}(Q)-\frac{b}{3}\left(Q: Q^{t}+2|Q|^{2}\right) .
$$

In the last term, we have used that $Q^{2}+Q Q^{t}+Q^{t} Q=Q_{i k} Q_{k j}+Q_{i k} Q_{j k}+Q_{k i} Q_{k j}$ hence

$$
\operatorname{tr}\left(Q^{2}+Q Q^{t}+Q^{t} Q\right)=Q: Q^{t}+2|Q|^{2}
$$

Therefore, taking the trace of system (1.3) and denoting $\phi=\operatorname{tr}(Q)$, the following $\phi$-PDE holds:

$$
\partial_{t} \phi+\boldsymbol{u} \cdot \nabla \phi+\gamma\left(-\varepsilon \Delta \phi+\left(a+c|Q|^{2}\right) \phi-\frac{b}{3}\left(Q: Q^{t}+2|Q|^{2}\right)\right)=0 .
$$

The main difficulties to obtain $\operatorname{tr}(Q(t))=0 \forall t \in(0, T)$ whenever $\operatorname{tr}\left(Q_{0}\right)=0$ (and $\operatorname{tr}\left(Q_{\Gamma}\right)=0$ in the Dirichlet case) are the terms in (7.58) depending on coefficient $b$ (which have no sign) and coefficient $a$ (which can be negative if $a<0$ ). In order to circumvent this problem, we consider the modified system

$$
D_{t} Q-S(\nabla \boldsymbol{u}, Q)=-\gamma \widetilde{H}(Q) \quad \text { in } \Omega \times(0, T) .
$$

obtained when $H$ defined in (1.6) is replaced by $\widetilde{H}$ defined in (1.16) with $\alpha(Q)$ defined in (1.17). Taking the trace of (7.59), we arrive at the modified $\phi$-equation:

$$
\partial_{t} \phi+\boldsymbol{u} \cdot \nabla \phi-\varepsilon \gamma \Delta \phi+\gamma \lambda c|Q|^{2} \phi=0
$$

Multiplying (7.60) by $\phi$ and integrating by parts, since the boundary term vanishes (recall that $\operatorname{tr}\left(Q_{\Gamma}\right)=0$ in the Dirichlet case) and $\gamma, \lambda$ and $c$ are positive, we arrive at:

$$
\frac{d}{d t}\|\phi\|_{L^{2}(\Omega)}^{2}+\varepsilon \gamma\|\nabla \phi\|_{L^{2}(\Omega)}^{2} \leq 0 \quad \Rightarrow \quad\|\phi(t)\|_{L^{2}(\Omega)}^{2} \leq\|\phi(0)\|_{L^{2}(\Omega)}^{2} .
$$


Finally, since $\phi(0)=0$, then $\phi(t)=0 \forall t$. As a consequence, $\operatorname{tr}(Q(t))=0$ for all $t \geq 0$. Using now that $\operatorname{tr}(Q)=0$, a unique new expression for $\alpha(Q)$ is deduced:

$$
\alpha(Q)=\frac{b}{9}\left(Q: Q^{t}+2|Q|^{2}\right) .
$$

Therefore $Q$ is also a weak solution for the modified $Q$-system using $\widetilde{H}=H+\alpha_{3}(Q) \mathbb{I}$ with

$$
\alpha_{3}(Q)=\frac{b}{9}\left(Q: Q^{t}+2|Q|^{2}\right)
$$

The extension of the main results of this paper (existence of weak solution, uniqueness criteria and maximum principle) when $H$ is replaced by $\widetilde{H}$ in the $Q$-system will be analyzed in B.

\subsection{Rewriting $S$ to obtain symmetry of $Q$}

Taking the transpose of tensorial system (1.3), the system verified by $Q^{t}$ is:

$$
D_{t} Q^{t}-S(\nabla \boldsymbol{u}, Q)^{t}+\gamma H(Q)^{t}=0
$$

Then, by using that $|Q|^{2}=\left|Q^{t}\right|^{2}$ and

$$
f(Q)^{t}=a Q^{t}-\frac{b}{3}\left(\left(Q^{t}\right)^{2}+Q^{t} Q+Q Q^{t}\right)+c\left|Q^{t}\right|^{2} Q^{t}=f\left(Q^{t}\right)
$$

one has

$$
H(Q)^{t}=-\varepsilon \Delta Q^{t}+(f(Q))^{t}=-\varepsilon \Delta Q^{t}+f\left(Q^{t}\right)=H\left(Q^{t}\right) .
$$

In a second step, we analyze the term $S(\nabla \boldsymbol{u}, Q)^{t}$ splitting $\nabla \boldsymbol{u}=\boldsymbol{D}+\boldsymbol{W}$, where $\boldsymbol{D}=$

$\frac{1}{2}\left(\nabla \boldsymbol{u}+(\nabla \boldsymbol{u})^{t}\right)$ is the symmetric part of $\nabla \boldsymbol{u}$ and $\boldsymbol{W}=\frac{1}{2}\left(\nabla \boldsymbol{u}-(\nabla \boldsymbol{u})^{t}\right)$ is the antisymmetric part of $\nabla \boldsymbol{u}$.

Owing to the linearity of $S(\cdot, Q)$,

$$
S(\nabla \boldsymbol{u}, Q)=S(\boldsymbol{D}, Q)+S(\boldsymbol{W}, Q)
$$

By using that $S(A, B)^{t}=B A^{t}-A^{t} B=-S\left(A^{t}, B\right)$, and the symmetry of $\boldsymbol{D}$ and the antisymmetry of $\boldsymbol{W}$, the transpose operator over the stretching term satisfies:

$$
S(\nabla \boldsymbol{u}, Q)^{t}=-S\left(\boldsymbol{D}, Q^{t}\right)+S\left(\boldsymbol{W}, Q^{t}\right) .
$$

Thus,

$$
S(\nabla \boldsymbol{u}, Q)-S(\nabla \boldsymbol{u}, Q)^{t}=S\left(\boldsymbol{D}, Q+Q^{t}\right)+S\left(\boldsymbol{W}, Q-Q^{t}\right) .
$$

Subtracting $(1.3)^{t}$ to $(1.3)$, one arrives at the following system verified by the "symmetry error" $E:=Q-Q^{t}:$

$$
D_{t} E-S\left(\boldsymbol{D}, Q+Q^{t}\right)-S(\boldsymbol{W}, E)+\gamma\left(-\varepsilon \Delta E+f(Q)-f\left(Q^{t}\right)\right)=0 .
$$


By using that $Q^{2}-\left(Q^{t}\right)^{2}=Q E+E Q^{t}$, the potential term is rewritten as:

$$
\begin{aligned}
f(Q)-f\left(Q^{t}\right) & =a E+c\left(|Q|^{2} Q-\left|Q^{t}\right|^{2} Q^{t}\right)-\frac{b}{3}\left[Q^{2}-\left(Q^{t}\right)^{2}\right] \\
& =a E+c|Q|^{2} E-\frac{b}{3}\left[Q E+E Q^{t}\right] .
\end{aligned}
$$

To avoid the influence of the term $S\left(\boldsymbol{D}, Q+Q^{t}\right)$ in (7.63), which is not zero when $Q$ is symmetric, we assume that the stretching term $S$ does not depend on $\boldsymbol{D}$, replacing $S(\nabla \boldsymbol{u}, Q)$ by $S(\boldsymbol{W}, Q)$ in the $Q$-system. Then, the $E$-system remains:

$$
D_{t} E-S(\boldsymbol{W}, E)+\gamma\left(-\varepsilon \Delta E+f(Q)-f\left(Q^{t}\right)\right)=0 .
$$

Multiplying (7.64) by $E$, integrating by parts (the boundary term vanishes using that $Q_{\Gamma}^{t}=Q_{\Gamma}$ in the Dirichlet case), using that $S(\boldsymbol{W}, E): E=0$ (see Lemma 3.1) and bounding:

$$
\int_{\Omega}\left(Q E+E Q^{t}\right): E d \boldsymbol{x} \leq 2\|Q\|_{\mathbb{L}^{\infty}(\Omega)}\|E\|_{\mathbb{L}^{2}(\Omega)}^{2} \leq C\|Q\|_{\mathbb{H}^{1}(\Omega)}^{1 / 2}\|Q\|_{\mathbb{H}^{2}(\Omega)}^{1 / 2}\|E\|_{\mathbb{L}^{2}(\Omega)}^{2},
$$

we obtain

$$
\begin{aligned}
\frac{1}{2} \frac{d}{d t}\|E\|_{\mathbb{L}^{2}(\Omega)}^{2} & +\gamma\left(\varepsilon\|\nabla E\|_{\mathbb{L}^{2}(\Omega)}^{2}+a\|E\|_{\mathbb{L}^{2}(\Omega)}^{2}+c \int_{\Omega}|Q|^{2}|E|^{2} d \boldsymbol{x}\right) \\
& \leq C b \gamma\|Q\|_{\mathbb{H}^{1}(\Omega)}^{1 / 2}\|Q\|_{\mathbb{H}^{2}(\Omega)}^{1 / 2}\|E\|_{\mathbb{L}^{2}(\Omega)}^{2}
\end{aligned}
$$

From Gronwall's Lemma, the following estimate is obtained:

$$
\begin{aligned}
& \|E(t, \cdot)\|_{\mathbb{L}^{2}(\Omega)}^{2} \leq\|E(0, \cdot)\|_{\mathbb{L}^{2}(\Omega)}^{2} \\
& \quad \times \exp \left[C b \gamma\|Q\|_{L^{\infty}\left(0, T ; \mathbb{H}^{1}(\Omega)\right)}^{1 / 2}\|Q\|_{L^{2}\left(0, T ; \mathbb{H}^{2}(\Omega)\right)}^{1 / 2} t^{3 / 4}-a \gamma t\right] .
\end{aligned}
$$

Assuming symmetric initial data $Q_{0}$, that means $E(0)=0$, we can conclude the symmetry of $Q(t)$, for each $t>0$, for any weak solution.

Remark 7.1 If the stretching term depending on $\boldsymbol{D}, S\left(\boldsymbol{D}, Q+Q^{t}\right)$, is not negligible in (7.63), then we could bound the term $\int_{\Omega} S\left(\boldsymbol{D}, Q+Q^{t}\right): E d \boldsymbol{x}$ arriving at:

$$
y^{\prime}(t) \leq C_{1} a(t) y(t)+C_{2} a(t) b(t),
$$

where $y(t)=\|E(t)\|_{\mathbb{L}^{2}(\Omega)}^{2}, a(t)=\|Q\|_{\mathbb{H}^{1}(\Omega)}^{1 / 2}\|Q\|_{\mathbb{H}^{2}(\Omega)}^{1 / 2}$ and $b(t)=\|\boldsymbol{D}(t)\|_{\mathbf{L}^{2}(\Omega)}^{2}$. Then $y(t) \neq 0$ in general even if $y(0)=0$, hence the symmetry of $Q(t)$ cannot be deduced.

A posteriori, by using the symmetry of any weak solution $Q$ (and $H$ ) of the modified system with $S(\boldsymbol{W}, Q)$ as stretching term, we have that $f(Q)=\widehat{f}(Q)$ and $S(\boldsymbol{W}, Q)=\widehat{S}(\boldsymbol{W}, Q)$ where $\widehat{f}(Q)$ and $\widehat{S}(\boldsymbol{W}, Q)$ are defined as:

$$
\widehat{f}(Q)=a Q-b Q^{2}+c|Q|^{2} Q
$$


and $\widehat{S}(\boldsymbol{W}, Q)$ is given in $(1.20)$. Therefore, any weak solution $Q$ is also a weak solution of the modified system with $\widehat{H}=-\varepsilon \Delta Q+\widehat{f}(Q)$ and (1.20) as stretching term.

The extension of the main results of this paper (existence of weak solution, uniqueness criteria and maximum principle) with $Q$ symmetric will be analyzed in C.

\subsection{A traceless and symmetric model}

Since the previous arguments for traceless and symmetry are independent they can act jointly. In fact, the symmetric argument is also true replacing $H(Q)$ by $\widetilde{H}(Q)=H+\alpha(Q) I$ with $\alpha(Q)$ defined in (1.17), because of $(\alpha(Q) I)^{t}=\alpha(Q) I$. Consequently, any weak solution of

the modified system replacing $H$ by $\widetilde{H}$ and $S(\nabla \boldsymbol{u}, Q)$ by $S(\boldsymbol{W}, Q)$ satisfies both constraints (symmetry and traceless).

In particular, considering the new expressions, where $f(Q)$ is replaced by $(7.65), S(\nabla \boldsymbol{u}, Q)$ by (1.20) and $H$ by $\widetilde{H}$ given in (1.16), then we derive the model (1.21) studied by Paicu and Zarnescu in [16].

Remark 7.2 To prove traceless of $Q$ in problem (1.21), inequality (7.61) must be replaced by

$$
\frac{d}{d t}\|\phi\|_{L^{2}(\Omega)}^{2}+\varepsilon \gamma\|\nabla \phi\|_{L^{2}(\Omega)}^{2}+a \gamma\|\phi(t)\|_{L^{2}(\Omega)}^{2} \leq 0
$$

with $\phi=\operatorname{tr}(Q)$, hence

$$
\|\phi(t)\|_{L^{2}(\Omega)}^{2} \leq\|\phi(0)\|_{L^{2}(\Omega)}^{2} \exp (-a \gamma t) .
$$

This last inequality introduces an exponential increasing in the bound of $\operatorname{tr}(Q(t))$ when $a<0$ and $\operatorname{tr}\left(Q_{0}\right)$ is not exactly zero.

\section{A Existence of Galerkin solutions without constraints.}

Let $\boldsymbol{V}^{m}=\left\langle\mathbf{w}^{1}, \ldots, \mathbf{w}^{m}\right\rangle$ and $\mathbb{M}^{n}=\left\langle\varphi^{1}, \ldots, \varphi^{n}\right\rangle$ be finite-dimensional subspaces associated to some regular basis $\left(\mathbf{w}^{i}\right)_{i}$ and $\left(\varphi^{j}\right)_{j}$ of the spaces

$$
\boldsymbol{V}=\left\{\overline{\boldsymbol{u}} \in \mathbf{H}_{0}^{1}(\Omega), \nabla \cdot \overline{\boldsymbol{u}}=0\right\}
$$

and

$$
\mathbb{M}=\left\{\boldsymbol{\varphi} \in \mathbb{H}^{2}(\Omega)+\text { homogeneous boundary conditions if necessary }\right\},
$$

respectively. In particular, we will describe the case of time-independent Dirichlet data for $Q$, because the Neumann case is less technical to treat. Then, let us introduce the lifting tensor $\widetilde{Q} \in \mathbb{H}^{2}(\Omega)$ as the (strong) solution of the elliptic problem

$$
-\Delta \widetilde{Q}=\mathbf{0} \quad \text { in } \Omega, \quad \widetilde{Q}=Q_{\Gamma} \quad \text { on } \Gamma .
$$


Now, we look for $\left(\boldsymbol{u}^{m}, Q^{n}\right)$ such that $\left(\boldsymbol{u}^{m}, Q^{n}-\widetilde{Q}\right) \in C^{1}\left([0, T] ; \boldsymbol{V}^{m} \times \mathbb{M}^{n}\right)$ solving:

$$
\left\{\begin{array}{rlr}
\left(\partial_{t} \boldsymbol{u}^{m}, \overline{\boldsymbol{u}}\right)+ & \left(\left(\boldsymbol{u}^{m} \cdot \nabla\right) \boldsymbol{u}^{m}, \overline{\boldsymbol{u}}\right)+\nu\left(\nabla \boldsymbol{u}^{m}, \nabla \overline{\boldsymbol{u}}\right) & \\
= & \left((\overline{\boldsymbol{u}} \cdot \nabla) Q^{n}, H^{n}\right)-\left(\sigma\left(H^{n}, Q^{n}\right), \nabla \overline{\boldsymbol{u}}\right), \quad \forall \overline{\boldsymbol{u}} \in \boldsymbol{V}^{m} \\
\left(\partial_{t} Q^{n}, \bar{Q}\right)+ & \left(\boldsymbol{u}^{m} \cdot \nabla Q^{n}, \bar{Q}\right)-\left(S\left(\nabla \boldsymbol{u}^{m}, Q^{n}\right), \bar{Q}\right) & \\
+ & \gamma\left(H^{n}, \bar{Q}\right)=0, & \\
\boldsymbol{u}^{m}(0)=\boldsymbol{u}_{0}, & Q^{n}(0)=Q_{0}, \quad \text { in } \Omega, &
\end{array} \quad \forall \bar{Q} \in \mathbb{M}^{n}\right.
$$

where

$$
H^{n}=P_{\mathbb{M}^{n}}\left(-\varepsilon \Delta Q^{n}+f\left(Q^{n}\right)\right)
$$

with $P_{\mathbb{M}^{n}}$ the projector from $\mathbb{L}^{2}(\Omega)$ onto $\mathbb{M}^{n}$. If the Galerkin functions $\left(\boldsymbol{u}^{m}, Q^{n}\right)$ are written as

$$
\boldsymbol{u}^{m}=\sum_{i=1}^{m} \eta_{i}(t) \mathbf{w}^{i}, \quad Q^{n}=\widetilde{Q}+\sum_{i=1}^{n} \phi_{i}(t) \varphi^{i}
$$

we can use the following identification operators:

$$
R^{\mathbf{V}^{\mathbf{m}}}: \boldsymbol{u}^{m}(t) \in \boldsymbol{V}^{m} \longrightarrow \mathcal{U}(t)=\left(\eta_{1}(t), \eta_{2}(t), \ldots, \eta_{m}(t)\right) \in \mathbb{R}^{m}
$$

and

$$
R^{\mathbb{M}^{n}}: Q^{n}(t) \in \widetilde{Q}+\mathbb{M}^{n} \longrightarrow \mathcal{Q}(t)=\left(\phi_{1}(t), \phi_{2}(t), \ldots, \phi_{n}(t)\right) \in \mathbb{R}^{n}
$$

Then, problem (A.67) can be rewritten as an ordinary differential system for $\mathcal{W}(t)=$ $(\mathcal{U}(t), \mathcal{Q}(t))^{t} \in C^{1}([0, T])^{m+n}$ that reads as:

$$
\left\{\begin{aligned}
\mathcal{W}^{\prime}(t)+\mathcal{A}(\mathcal{W}(t)) & =0 \\
\mathcal{W}(0) & =\left(R^{\mathbf{V}^{\mathbf{m}}}\left(P_{\boldsymbol{V}^{m}}\left(\boldsymbol{u}_{0}\right)\right), R^{\mathbb{M}^{n}}\left(\widetilde{Q}+P_{\mathbb{M}^{n}}\left(Q_{0}-\widetilde{Q}\right)\right)\right)
\end{aligned}\right.
$$

Note that in the case of time-dependent Dirichlet data for $Q$ a non-zero source term appears at the right-hand side of previous system, depending on $\partial_{t} \widetilde{Q}$.

If the operator $\mathcal{A}$ is continuous, then we can conclude the existence of a solution $\mathcal{W} \in$ $C^{1}\left(\left[0, T^{*}\right)\right)$ where either $T^{*}=+\infty$ or $T^{*}<+\infty$ and $\lim _{t \uparrow T^{*}}\|\mathcal{W}(t)\|_{\mathbb{R}^{m+n}}=+\infty$. In fact, we are going to prove that the solution $\mathcal{W}(t)$ is defined in $[0,+\infty)$. For this aim, we consider $(\bar{u}, \bar{Q})=\left(\boldsymbol{u}^{m}, H^{n}\right) \in \boldsymbol{V}^{m} \times \mathbb{M}^{n}$ in (A.67), obtaining an energy law as (3.26) for $\left(\boldsymbol{u}^{m}, Q^{n}\right)$ and the global in time weak estimates given in (1.11). Therefore, taking into account the equivalence of the norms in finite-dimensional spaces, the solution $\left(\boldsymbol{u}^{m}, Q^{n}\right)$ is defined in the whole $[0,+\infty)$.

It remains to prove the continuity of $\mathcal{A}$. Observe that:

$$
\mathcal{A}: \mathcal{W}(t) \in \mathbb{R}^{m+n} \longrightarrow \mathcal{A}(\mathcal{W}(t)) \in \mathbb{R}^{m+n}
$$


is defined as

$$
\mathcal{A}(\mathcal{W}(t))=\mathcal{B}(\mathcal{W}(t))+\left(\begin{array}{l}
\mathcal{C}_{1}(\mathcal{Q}(t)) \\
\mathcal{C}_{2}(\mathcal{Q}(t))
\end{array}\right)
$$

with $\mathcal{B}(\cdot)$ a polinomial function,

$$
\mathcal{C}_{1}(\mathcal{Q}(t))_{k}=\mathcal{Q}_{i}(t) \mathcal{D}(\mathcal{Q}(t))_{j}\left(\sigma\left(\varphi^{j}, \varphi^{i}\right), \nabla \mathbf{w}^{k}\right)-\mathcal{Q}_{i}(t) \mathcal{D}(\mathcal{Q}(t))_{j}\left(\left(\mathbf{w}^{k} \cdot \nabla\right) \varphi^{i}, \varphi^{j}\right)
$$

and

$$
\mathcal{C}_{2}(\mathcal{Q}(t))_{l}=\mathcal{D}(\mathcal{Q}(t))_{j}\left(\varphi^{j}, \varphi^{l}\right)
$$

where

$$
\mathcal{D}(\mathcal{Q}(t))=R^{\mathbb{M}^{n}} \circ P_{\mathbb{M}^{n}} \circ \Phi \circ\left(R^{\mathbb{M}^{n}}\right)^{-1}(\mathcal{Q}(t))
$$

with $R^{\mathbb{M}^{n}}$ defined in (A.68) and $\Phi$ defined by:

$$
\Phi: Q^{n} \in \mathbb{M}^{n} \subset \mathbb{H}^{2}(\Omega) \longrightarrow-\varepsilon \Delta Q^{n}+f\left(Q^{n}\right) \in \mathbb{L}^{2}(\Omega)
$$

From (A.69), owing to the Projection Theorem $\left(\left\|P_{\mathbb{M}^{n}}\right\|_{\mathcal{L}\left(\mathbb{L}^{2}(\Omega), \mathbb{L}^{2}(\Omega)\right)} \leq 1\right)$ and the properties of $R^{\mathbb{M}^{n}}$, it suffices to prove the continuity of $\Phi$, which is an easy consequence of the properties of the Laplace operator $-\Delta Q$ and the polinomial function $f(Q)$.

\section{B Results for the traceless model}

Here, we will extend the main results of this paper, namely existence of weak solutions, uniqueness criteria and maximum principle, to the modified $Q$-tensor model, replacing (in the $Q$-system (1.3) and also in the $\boldsymbol{u}$-system $(1.2)) H(Q)$ by $\widetilde{H}(Q)=H(Q)+\alpha(Q) \mathbb{I}$ with $\alpha(Q)$ given by (1.17).

By simplicity, we will only detail the main modifications in the corresponding proofs.

\section{B.1 Existence of weak solutions.}

It suffices to arrive at a modified energy law (see (B.70) below). From (1.16) and the expression describing the tensor $\sigma(\cdot, \cdot)$, one has $\sigma(H, Q)=\sigma(\widetilde{H}, Q)$.

We only treat the terms related to $H(Q)$ and study the fact of replacing it by $\widetilde{H}(Q)$. By using (2.22), the tensor $\tau$ can be rewritten as:

$$
\begin{aligned}
(\nabla \cdot \tau)_{i} & =\widetilde{H}: \partial_{i} Q-\partial_{i}\left(F(Q)+\frac{\varepsilon}{2}|\nabla Q|^{2}\right)-\alpha(Q) I: \partial_{i} Q \\
& =\widetilde{H}: \partial_{i} Q-\partial_{i}\left(F(Q)+\frac{\varepsilon}{2}|\nabla Q|^{2}\right)-\alpha(Q) \partial_{i}(\operatorname{tr}(Q))
\end{aligned}
$$

By using that $\operatorname{tr}(Q)=0$, one has:

$$
(\nabla \cdot \tau, \boldsymbol{u})=((\boldsymbol{u} \cdot \nabla) Q, \widetilde{H})
$$


Moreover, using again $\partial_{t}(\operatorname{tr}(Q))=0$,

$$
\left(\partial_{t} Q, \widetilde{H}\right)=\frac{d}{d t} \mathcal{E}(Q)+\left(\partial_{t}(\operatorname{tr}(Q)), \alpha(Q)\right)=\frac{d}{d t} \mathcal{E}(Q) .
$$

Therefore, we arrive at the following energy equality similar to (3.26):

$$
\frac{d}{d t}\left(\frac{1}{2}\|\boldsymbol{u}\|_{\mathbf{L}^{2}(\Omega)}^{2}+\mathcal{E}(Q)\right)+\nu\|\nabla \boldsymbol{u}\|_{\mathbf{L}^{2}(\Omega)}^{2}+\gamma\|\widetilde{H}\|_{\mathbb{L}^{2}(\Omega)}^{2}=0
$$

From now on, the existence argument follows in a similar manner.

\section{B.2 Uniqueness criteria}

When considering $\widetilde{f}(Q)=f(Q)+\alpha(Q) \mathbb{I}$ with $\alpha(Q)$ given in (1.17), we must measure the bound for $\left\|\alpha\left(Q_{1}\right)-\alpha\left(Q_{2}\right)\right\|_{\mathbb{L}^{2}(\Omega)}$. Observe that

$$
\left\|\alpha\left(Q_{1}\right)-\alpha\left(Q_{2}\right)\right\|_{\mathbb{L}^{2}(\Omega)} \leq C\left\|Q_{1}^{2}-Q_{2}^{2}\right\|_{\mathbb{L}^{2}(\Omega)}
$$

and $\left\|Q_{1}^{2}-Q_{2}^{2}\right\|_{\mathbb{L}^{2}(\Omega)}$ can be bounded as the term $\left\|F_{e}^{\prime}\left(Q_{1}\right)-F_{e}^{\prime}\left(Q_{2}\right)\right\|_{\mathbb{L}^{2}(\Omega)}$ in the proof of Theorem 1.7 .

\section{B.3 Maximum principle}

It is only necessary to study the effect of the new term $\widetilde{H}(Q)$ in the computation:

$$
\widetilde{H}(Q): Q=H(Q): Q+\alpha(Q) \mathbb{I}: Q=H(Q): Q+\alpha(Q) \operatorname{tr}(Q)=H(Q): Q
$$

owing to $\operatorname{tr}(Q)=0$. Therefore, we can extend the maximum principle given in Theorem 1.9 to the modified traceless model.

\section{Results for the model with symmetry}

In this case, we replace the stretching term $S(\nabla \boldsymbol{u}, Q)$ by $S(\boldsymbol{W}, Q)$ in the $Q$-system (1.3). As in $\mathrm{B}$, we only analyze the modifications to obtain the main results of this paper.

\section{C.1 Existence of weak solutions}

Since now $Q$ and $H$ are symmetric then $\sigma(H, Q)$ is antisymmetric. Therefore, replacing $S(\nabla \boldsymbol{u}, Q)$ by $\widehat{S}(\boldsymbol{W}, Q)$ (defined in $(1.20)$ ), we obtain:

$$
\widehat{S}(\boldsymbol{W}, Q): H=\sigma(H, Q): \boldsymbol{W}=\sigma(H, Q): \nabla \boldsymbol{u}
$$

This equality implies that the energy law in the modified symmetric problem also holds. 


\section{C.2 Uniqueness criteria}

Replacing $S(\nabla \boldsymbol{u}, Q)$ by $\widehat{S}(\boldsymbol{W}, Q)$, we have to change (5.46) by:

$$
\left.\left(\sigma\left(H_{c}, Q_{1}\right), \boldsymbol{W}\right)\right)-\left(\widehat{S}\left(\boldsymbol{W}, Q_{1}\right), H_{c}\right)=0
$$

that vanishes because $\widehat{S}\left(\boldsymbol{W}, Q_{1}\right): H_{c}=\boldsymbol{W}: \sigma\left(H_{c}, Q_{1}\right)$. Moreover, estimates of $I_{4}, I_{5}$ and $I_{8}$-terms (depending on $\nabla \boldsymbol{u}$ ) are similar whether $I_{4}, I_{5}$ and $I_{8}$ depend on $\boldsymbol{W}$.

\section{C.3 Maximum principle}

Owing to Lemma 3.1, $\widehat{S}(\boldsymbol{W}, Q): Q=0$ hence the maximum principle argument is again true.

\section{Traceless and/or symmetric Galerkin approximations}

Any weak solution satisfies the $Q$-system point-wisely, hence the arguments to obtain symmetry or traceless for $Q$ can be made a posteriori, directly from the weak solutions. Consequently, it is not necessary to impose these constraints over the Galerkin approximation $Q^{n}$.

However, it is possible to conserve the symmetry and/or traceless for the Galerkin approximation $Q^{n}$. Fistly, since these constraints and the lifting problem (A.66) are linear then symmetry and/or traceless are extended from $Q_{\Gamma}$ to $\widetilde{Q}$, in the Dirichlet case for $Q$. On the other hand, we rewrite (A.67) $)_{3}$ as:

$$
\begin{aligned}
\left(\partial_{t} Q^{n}, \bar{Q}\right) & +\left(\boldsymbol{u}^{m} \cdot \nabla Q^{n}, \bar{Q}\right)-\left(S\left(\nabla \boldsymbol{u}^{m}, Q^{n}\right), \bar{Q}\right) \\
& +\varepsilon \gamma\left(\nabla Q^{n}, \nabla \bar{Q}\right)+\gamma\left(f\left(Q^{n}\right), \bar{Q}\right)=0, \quad \forall \bar{Q} \in \mathbb{M}^{n}
\end{aligned}
$$

Then, some conditions for the space $\mathbb{M}^{n}$ must be imposed:

- To deduce traceless, we rewrite (D.71) replacing $f\left(Q^{n}\right)$ by $f\left(Q^{n}\right)+\alpha\left(Q^{n}\right) \mathbb{I}$, for $\alpha(Q)$ defined in (1.17). Then, we take $\operatorname{tr}\left(Q^{n}\right) \mathbb{I} \in \mathbb{M}^{n}$ as test function in (D.71), assuming that the space $\mathbb{M}^{n}$ is defined for the same scalar space in each diagonal component, that is, for each $i=1,2,3\left(\mathbb{M}^{n}\right)_{i i}=M^{n}$ with $M^{n}$ a scalar space. Thus, we obtain (7.61) hence the traceless property for $Q^{n}$ can be deduced.

- To obtain symmetry, we replace $S\left(\nabla \boldsymbol{u}^{m}, Q^{n}\right)$ by $\widehat{S}\left(\boldsymbol{W}\left(\boldsymbol{u}^{m}\right), Q^{n}\right)$ defined in (1.20). Taking into account that transposing the $Q$-system is equivalent to take $\bar{Q}=\bar{Q}^{t}$ in (D.71), the symmetry of $Q^{n}$ can be proved because:

$$
\left(Q^{n} \text {-system- }\left(Q^{n} \text {-system }\right)^{t}, Q^{n}-\left(Q^{n}\right)^{t}\right)
$$

is equivalent to

$$
\left(Q^{n} \text {-system, } 2\left(Q^{n}-\left(Q^{n}\right)^{t}\right)\right)
$$


Therefore, this last computation can be done imposing $\left(\mathbb{M}^{n}\right)_{i j}=\left(\mathbb{M}^{n}\right)_{j i}$ for $i \neq j$ which implies that $\left(Q^{n}\right)^{t} \in \mathbb{M}^{n}$.

\section{Acknowledgements}

The authors have been partially financed by the project MTM2012-32325.

\section{References}

[1] H. Abels, G. Dolzmann, and Y. Liu. Strong solutions for the beris-edwards model for nematic liquid crystals with homogeneous dirichlet boundary conditions. arxiv 1312.5988, 2013.

[2] H. Abels, G. Dolzmann, and Y. Liu. Well-posedness of a fully-coupled navier-stokes $/ q$ tensor system with inhomogeneous boundary data. arXiv:1311.3443, 2013.

[3] Antony N. Beris and Brian J. Edwards. Thermodynamics of flowing systems with internal microstructure, volume 36 of Oxford Engineering Science Series. The Clarendon Press, Oxford University Press, New York, 1994. Oxford Science Publications.

[4] Luigi C. Berselli. On a regularity criterion for the solutions to the 3D Navier-Stokes equations. Differential Integral Equations, 15(9):1129-1137, 2002.

[5] Cecilia Cavaterra and Elisabetta Rocca. On a 3D isothermal model for nematic liquid crystals accounting for stretching terms. Z. Angew. Math. Phys., 64(1):69-82, 2013.

[6] S. Chandrasekhar. Liquid Crystals. International Series of Monographs on Physics. Cambridge University Press, Cambridge, 1992 (2nd ed. ).

[7] Blanca Climent-Ezquerra, Francisco Guillén-González, and Marko Rojas-Medar. Reproductivity for a nematic liquid crystal model. Z. Angew. Math. Phys., 57(6):984-998, 2006.

[8] P.G. de Gennes and J. Prost. The Physics of Liquid Crystals. International Series of Monographs on Physics. Clarendon Press, 1995.

[9] Colin Denniston, Enzo Orlandini, and J. M. Yeomans. Lattice boltzmann simulations of liquid crystal hydrodynamics. Phys. Rev. E, 63:056702, Apr 2001.

[10] Jishan Fan and Tohru Ozawa. Regularity criteria for a coupled Navier-Stokes and $Q$-tensor system. Int. J. Anal., pages Art. ID 718173, 5, 2013.

[11] F. Guillén-González and M. A. Rodríguez-Bellido. Weak time regularity and uniqueness for a $Q$-tensor model. To appear in SIMA. 
[12] F. Guillén-González, M. A. Rodríguez-Bellido, and M. A. Rojas-Medar. Sufficient conditions for regularity and uniqueness of a 3D nematic liquid crystal model. Math. Nachr., 282(6):846-867, 2009.

[13] F. M. Leslie. Some constitutive equations for liquid crystals. Arch. Rational Mech. Anal., 28(4):265-283, 1968.

[14] Apala Majumdar and Arghir Zarnescu. Landau-De Gennes theory of nematic liquid crystals: the Oseen-Frank limit and beyond. Arch. Ration. Mech. Anal., 196(1):227-280, 2010.

[15] Marius Paicu and Arghir Zarnescu. Global existence and regularity for the full coupled Navier-Stokes and Q-tensor system. SIAM J. Math. Anal., 43(5):2009-2049, 2011.

[16] Marius Paicu and Arghir Zarnescu. Energy dissipation and regularity for a coupled NavierStokes and Q-tensor system. Arch. Ration. Mech. Anal., 203(1):45-67, 2012.

[17] Jacques Simon. Compact sets in the space $L^{p}(0, T ; B)$. Ann. Mat. Pura Appl. (4), 146:6596, 1987.

[18] Huan Sun and Chun Liu. On energetic variational approaches in modeling the nematic liquid crystal flows. Discrete Contin. Dyn. Syst., 23(1-2):455-475, 2009.

[19] Géza Tóth, Colin Denniston, and J. M. Yeomans. Hydrodynamics of domain growth in nematic liquid crystals. Phys. Rev. E, 67:051705, May 2003.

[20] Hao $\mathrm{Wu}$, Xiang Xu, and Chun Liu. Asymptotic behavior for a nematic liquid crystal model with different kinematic transport properties. Calc. Var. Partial Differential Equations, $45(3-4): 319-345,2012$.

[21] Arghir Zarnescu. Topics in the Q-tensor theory of liquid crystals. In Topics in mathematical modeling and analysis, volume 7 of Jindurich Neucas Cent. Math. Model. Lect. Notes, pages 187-252. Matfyzpress, Prague, 2012. 